\title{
The $i^{6} \mathrm{~A} 37$ tRNA modification is essential for proper decoding of UUX-Leucine codons during rpoS and iraP translation
}

\author{
JOSEPH I. AUBEE, ${ }^{1}$ MORENIKE OLU, ${ }^{1,2}$ and KARL M. THOMPSON ${ }^{1}$ \\ ${ }^{1}$ Department of Microbiology, College of Medicine, Howard University, Washington, DC 20059, USA \\ ${ }^{2}$ Department of Biology, Howard University, Washington, DC 20059, USA
}

\begin{abstract}
The translation of $r p o S\left(\sigma^{S}\right)$, the general stress/stationary phase sigma factor, is tightly regulated at the post-transcriptional level by several factors via mechanisms that are not clearly defined. One of these factors is MiaA, the enzyme necessary for the first step in the $N^{6}$-isopentyl-2-thiomethyladenosinemethyladenosine $37\left(\mathrm{~ms}^{2} \mathrm{i}^{6} \mathrm{~A} 37\right)$ tRNA modification. We tested the hypothesis that an elevated UUX-Leucine/total leucine codon ratio can be used to identify transcripts whose translation would be sensitive to loss of the MiaA-dependent modification. We identified iraP as another candidate MiaA-sensitive gene, based on the UUX-Leucine/ total leucine codon ratio. An iraP-lacZ fusion was significantly decreased in the absence of MiaA, consistent with our predictive model. To determine the role of MiaA in UUX-Leucine decoding in $r p o S$ and iraP, we measured $\beta$-galactosidasespecific activity of $\mathrm{miaA}^{-}$rpoS and iraP translational fusions upon overexpression of leucine tRNAs. We observed suppression of the MiaA effect on rpoS, and not iraP, via overexpression of tRNA ${ }^{\text {LeuX }}$ but not tRNA ${ }^{\text {LeuZ }}$. We also tested the hypothesis that the MiaA requirement for $r p o S$ and iraP translation is due to decoding of UUX-Leucine codons within the $r p o S$ and iraP transcripts, respectively. We observed a partial suppression of the MiaA requirement for rpoS and iraP translational fusions containing one or both UUX-Leucine codons removed. Taken together, this suggests that MiaA is necessary for rpoS and iraP translation through proper decoding of UUX-Leucine codons and that $r p o S$ and iraP mRNAs are both modification tunable transcripts (MoTTs) via the presence of the modification.
\end{abstract}

Keywords: tRNA modification; translation; MiaA; leu codon; RpoS

\section{INTRODUCTION}

Transfer RNA (tRNA) modifications play a critical role in the promotion of translation fidelity (Urbonavicius et al. 2001). The absence of tRNA modifications is known to increase the frequency of translational frameshifting (Björk et al. 1999; Urbonavicius et al. 2001, 2003). There are a host of tRNA modifications that are most crucial in translational frameshift suppression and the majority of them reside within the anticodon stem-loop (ASL), flanking the anticodon at positions 34 and 37, including 5-methyluridine ( $\left.\mathrm{m}^{5} \mathrm{U} 34\right), 5$ methylcytidine $\left(\mathrm{m}^{5} \mathrm{C} 34\right)$, and $N^{6}$-isopentenyladenosine (i $\left.{ }^{6} \mathrm{~A} 37\right)$ (Endres et al. 2015). Certain tRNA modifications act in a regulatory manner on physiological circuits within the cell, such as DNA damage and oxidative stress in eukaryotic cells (Begley et al. 2007; Chan et al. 2010, 2012; Patil et al. 2012; Lamichhane et al. 2013a). There is limited information on the regulatory role of tRNA modifications in bacterial cells. We previously identified an important role for the Escherichia coli $\mathrm{ms}^{2} \mathrm{i}^{6} \mathrm{~A} 37$ synthesis enzyme,

Corresponding author: karl.thompson@howard.edu

Article published online ahead of print. Article and publication date are at http://www.rnajournal.org/cgi/doi/10.1261/rna.053165.115.
MiaA, in the translation of rpoS mRNA (Thompson and Gottesman 2014).

RpoS $\left(\right.$ or $\sigma^{\mathrm{S}}$ ) is the stationary phase/general stress response alternative sigma factor necessary for the adaptation of bacterial cells to the stationary phase environment (HenggeAronis 1993, 1996). RpoS contributes to stationary phase homeostasis by initiating the transcription of a large subset of genes that respond to limiting nutrients and increased exposure to reactive oxygen intermediates (Hengge-Aronis 1993; Tanaka et al. 1993). Since RpoS levels are important for the stationary phase stress response, RpoS levels are tightly regulated at the transcriptional level and at post-transcriptional levels (Lange et al. 1995; Brown and Elliot 1996; Hirsch and Elliott 2002). RpoS translation is stimulated by the three Hfq-dependent small regulatory RNAs: DsrA, RprA, and ArcZ in response to different environmental signals (Sledjeski et al. 1996; Majdalani et al. 1998, 2002; Mandin and Gottesman 2010). RpoS is also regulated at the level of protein stability by the ATP-dependent protease ClpXP,

(C) 2016 Aubee et al. This article is distributed exclusively by the RNA Society for the first 12 months after the full-issue publication date (see http:// rnajournal.cshlp.org/site/misc/terms.xhtml). After 12 months, it is available under a Creative Commons License (Attribution-NonCommercial 4.0 International), as described at http://creativecommons.org/licenses/by-nc/4.0/. 
with the assistance of RssB, which serves as an adaptor protein (Gottesman 1996; Zhou and Gottesman 1998). There are three anti-adaptor proteins, IraP, IraD, and IraM, which all prevent RssB interaction with RpoS under different conditions and increase RpoS stability as a consequence (Bougdour et al. 2006, 2008).

In addition to stimulation of translational initiation by several small regulatory RNAs and regulation of RpoS stability, translation of the rpoS open reading frame (ORF) is also regulated by cis and trans acting elements. For example, SsrA is necessary for $r p o S$ translation through a mechanism that is not completely understood (Ranquet and Gottesman 2007). The presence of rare codons within the rpoS ORF also positively contributes to mRNA stability by decreasing degradation by RNaseE (Kolmsee and Hengge 2011). Additionally, AceE, a subunit of pyruvate dehydrogenase, also influences rpoS ORF translation via an undefined mechanism (Battesti et al. 2015). Finally, the MiaA tRNA modification enzyme is required for efficient translation of the $r p o S$ ORF translation (Thompson and Gottesman 2014).

$\mathrm{MiaA}$ is required for the first step in the formation of $\mathrm{ms}^{2} \mathrm{i}^{6} \mathrm{~A} 37$ modification on tRNAs that read UUX codons (Bartz et al. 1970). Specifically, MiaA catalyzes the addition of the isopentyl group $\left(i^{6}\right)$ to A37 of these tRNAs (Bartz et al. 1970). MiaB then catalyzes the addition of a methylthio group $\left(\mathrm{ms}^{2}\right)$ to complete the $\mathrm{ms}^{2} \mathrm{i}^{6} \mathrm{~A} 37$ tRNA modification (Vold et al. 1979; Esberg et al. 1999). While MiaB is necessary for completing the $\mathrm{ms}^{2}{ }^{6}{ }^{6} \mathrm{~A} 37 \mathrm{tRNA}$ modification, our initial analysis of the effect of tRNA modifications on rpoS suggested that MiaB was not necessary for full rpoS expression (Thompson and Gottesman 2014). Therefore, this work is focused on characterizing the MiaA effect on rpos. MiaA is necessary for expression of genes involved in the biosynthesis of amino acids in Escherichia coli and Salmonella enterica subspecies enterica servar Typhimurium, including tryptophan and phenylalanine in E. coli as well as leucine in S. typhimurium (Gowrishankar and Pittard 1982; Blum 1988). The MiaA requirement for expression of the tryptophan and phenylalanine operons is due to its role in modulating transcriptional attenuation (Landick et al. 1990; Pages and Buckingham 1990). We hypothesized that leucine codon usage may play a role in the requirement for MiaA in the efficient translation of rpoS, due to use of UUX-Leucine rather than CXX-Leucine codons within the rpos ORF (Thompson and Gottesman 2014).

Here, we test this hypothesis, both extending our previous tests with rpoS to another UUX-rich gene, iraP. We demonstrate the ability to identify putative $\mathrm{ms}^{2} i^{6} \mathrm{~A} 37$ modification tunable transcripts (MoTTs), via MiaA sensitivity, for a given gene based on UUX-Leucine codon usage for that gene (Endres et al. 2015). We hypothesize that genes for ORFs with high UUX Leucine codon usage, or HULC proteins, defined as having a UUX-Leucine codon usage ratio $>0.22$, likely have $i^{6} \mathrm{~A} 37$ MoTTs. We show that iraP has a similar enrichment ratio for UUX-Leucine codons as rpoS and that its translation requires the $i^{6} \mathrm{~A} 37$ tRNA modification catalyzed by MiaA. Furthermore, we demonstrate that UUX-leu to CUX-leu codon substitutions within rpoS and iraP can suppress the effects of $\triangle$ miaA mutants on the translation of these ORFs, suggesting that MiaA is required for rpoS and iraP translation, at least in part, for efficient decoding of UUX-Leucine codons in these two genes.

\section{RESULTS}

\section{Replacing MiaA-sensitive leucine codons (UUGs and UUAs) with MiaA-insensitive leucine codons (CUUs and CUCs) partially suppresses the MiaA requirement for rpoS synthesis}

We previously used an arabinose-inducible rpoS990-lacZ translational fusion to demonstrate the role of the MiaA-catalyzed $i^{6} \mathrm{~A}$ modification on rpoS synthesis (Thompson and Gottesman 2014). The $\mathrm{P}_{B A D^{-}}$rpoS990-lac $Z$ translation fusion contains the entire rpoS ORF, except for the termination codon; the small-RNA responsive 5' UTR of $r p o S$ and the native promoter are replaced by the araBAD promoter $\left(\mathrm{P}_{B A D}\right)$ (Fig. 1B; Supplemental Table S1; Thompson and Gottesman 2014). All $\mathrm{P}_{B A D^{-}}$rpoS990-lacZ translation fusion experiments were executed in an $r s s B^{-}$background to rule out effects on rpos degradation.

We previously hypothesized that the greater than expected ratio of MiaA-sensitive leucine codon to total leucine codon usage within the rpoS ORF suggested that MiaA may function to ensure proper decoding of these UUX-Leucine codons within rpoS (Thompson and Gottesman 2014). To test this hypothesis, we constructed a $\mathrm{P}_{B A D^{-}}$rpoS990-lacZ translational fusion in which the first and wobble position of all MiaA-sensitive leucine codons (UUAs and UUGs) in rpos were changed to create MiaA-insensitive leucine codons (CUUs and CUCs), creating a series of silent leucine mutations throughout the rpoS990 region of the translational fusion (Table 1, leu*3; Fig. 1B). The rationale behind the selection of CUU and CUC codons is that they are present at frequencies similar to UUA and UUG codons (http://www.sci.sdsu. edu/ smaloy/MicrobialGenetics/topics/in-vitro-genetics/ codon-usage.html). There are six codons that code for leucine, UUR, and CUX. The average frequency of decoding for leucine by CUU and CUC codons are 0.10, which is similar to the 0.11 frequency seen with UUA and UUG codons. In contrast, CUA and CUG Leu codons have average frequencies of 0.03 and 0.55 , respectively.

We then measured the $\beta$-galactosidase-specific activity (as defined in Materials and Methods) as the slope of $\mathrm{OD}_{420} /$ OD600 of the wild-type and leu*3 fusions in $\mathrm{rss}^{-} \mathrm{miaA}^{+}$ and $r s s B^{-}$mia $^{-}$genetic backgrounds (Fig. 1A). There was an exponential increase in the $\beta$-galactosidase-specific activity of both the wild-type $\mathrm{P}_{B A D^{-}}$rpoS990-lacZ and $\mathrm{P}_{B A D^{-}}$ rpoS990(leu*3)-lacZ translational fusions in the presence of MiaA (Fig. 1A). As previously reported, the absence of 
A

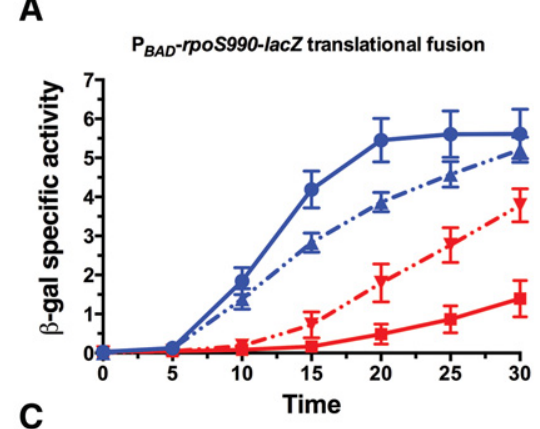

C

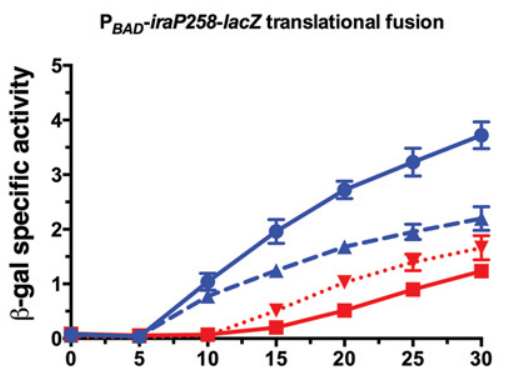

B

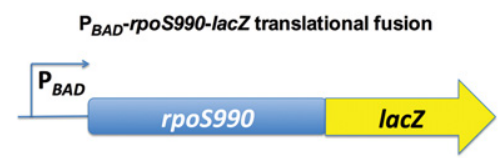

$-\operatorname{mia} A+$
$-\operatorname{mia} A-$

- $\operatorname{mia} A+\left(l e u^{\star} 3\right)$

$\rightarrow$ miaA- $\left(l e{ }^{\star} 3\right)$

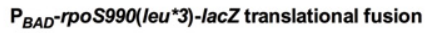

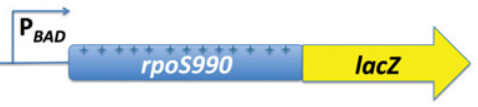

D

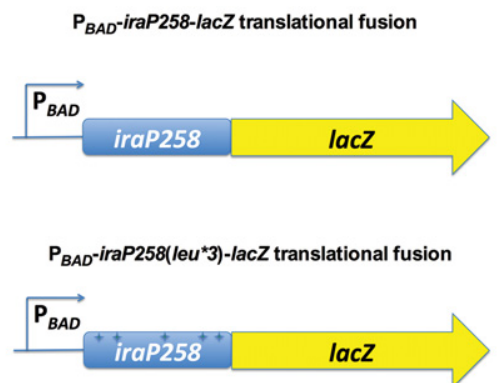

FIGURE 1. Translation of $l e u^{*} 3$ alleles of $r p o S$ and $i r a P$ in the absence of $\mathrm{ms}^{2} \mathrm{i}^{6} \mathrm{~A} 37$ tRNA modification. $(A)$ The $r s s B^{-}$miaA $^{+}\left(\mathrm{KMT}_{3} 3001\right)$ and $r s s B^{-}$ miaA $^{-}$(KMT33002) $\mathrm{P}_{B A D^{-}}$rpoS990(leu*3)-lacZ translational fusion strains, as well as rssB $B^{-}$miaA $^{+}$(KMT30003) and rssB $B^{-}$miaA $A^{-}$(KMT30011A) $\mathrm{P}_{B A D^{-}}$-rpoS990-lac $Z$ translational fusion strains, were assayed for $\beta$-galactosidase-specific activity following arabinose induction. $\beta$-galactosidase-specific activity is defined as the slope of $\mathrm{OD}_{420}$ of the collected sample cell lysate divided by the $\mathrm{OD}_{600}$ of the collected sample of the culture, as described in Materials and Methods. Each value represents the mean of at least three replicate experiments; the error bars represent the standard error of the mean (SEM). (B) Schematic comparison of the $\mathrm{P}_{B A D^{-}}$rpoS990-lacZ and $\mathrm{P}_{B A D^{-}} r p o S 990($ leu*3)-lacZ translational fusions used in this experiment. The stars within the $\mathrm{P}_{B A D^{-}} r p o S 990\left(l e u^{*} 3\right)$-lac $Z$ translational fusion represent silent leucine mutations where rare leucine codons (UUA and UUG) were changed to abundant leucine codons (CUU or CUC). (C) The miaA ${ }^{+}$(KMT45000) and miaA ${ }^{-}$(KMT45002) $\mathrm{P}_{B A D^{-}}$iraP258(leu*3)-lacZ translational

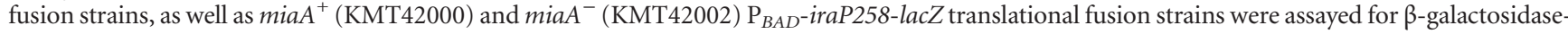
specific activity following arabinose induction. Each value represents the mean of at least three replicate experiments; the error bars represent the standard error of the mean (SEM). (D) Schematic comparison of the $\mathrm{P}_{B A D}$-iraP258-lacZ and $\mathrm{P}_{B A D}$-iraP258(leu*3)-lacZ translational fusions used in this experiment. The green stars within the $\mathrm{P}_{B A D}$-iraP258(leu*3)-lacZ translational fusion represent silent leucine mutations where rare leucine codons (UUA and UUG) were changed to abundant leucine codons (CUU or CUC). Each value represents the mean of at least three replicate experiments; the error bars represent the standard error of the mean (SEM).

MiaA resulted in severely defective induction of the $\mathrm{P}_{B A D^{-}}$ rpoS990-lacZ in comparison to the miaA ${ }^{+}$strain (Fig. 1A). Specifically, $25 \mathrm{~min}$ after arabinose induction $\left(t_{25}\right)$, there was a fivefold decrease in $\mathrm{P}_{B A D^{-}}$- ppoS990-lac $Z$ expression in the absence of MiaA (Fig. 1A). The induction of the $\mathrm{P}_{B A D^{-}}$ rpoS990(leu*3)-lacZ translational fusion was not as defective in the absence of MiaA in comparison to the wild type (1.5fold decrease in $\mathrm{P}_{B A D^{-}}$rpoS990[leu*3]-lacZ) expression in the absence of MiaA at $t_{25}$ (compared to fivefold in the wild-type fusion). This suggests that the presence of UUX-Leucine codons, which are sensitive to MiaA-modified leucine tRNAs, contribute to the requirement of MiaA for full rpoS synthesis. However, the fact that the removal of the UUX-Leucine codons did not result in full suppression of the MiaA requirement for rpoS synthesis suggests that MiaA does more for rpoS translation than just ensuring that UUX-Leucine tRNAs properly recognize their cognate codons. MiaA-sensitive codons also consist of UUX phenylalanine, UCX serine, UAX tyrosine, UGX cysteine, and UGG tryptophan. It is possible that these codons may not be sufficiently decoded in the absence of MiaA as well. Also, the expression from the $\mathrm{miaA}^{+}$
$\mathrm{P}_{B A D^{-}}$rpoS990(leu*3)-lacZ translational fusion was slightly decreased in comparison to the miaA ${ }^{+} \mathrm{P}_{B A D^{-}}$PpoS990-lacZ, suggesting that the UUX-Leucine codons are required for optimal rpoS translation in the presence of MiaA.

TABLE 1. Silent leucine mutations in the rpoS leu* alleles

\begin{tabular}{lcc}
\hline Amino acid & Original codon & Leu* \\
\hline Leucine 11 & UUA & CUU \\
Leucine 30 & UUA & CUU \\
Leucine 40 & UUG & CUC \\
Leucine 46 & UUA & CUU \\
Leucine 55 & UUG & CUC \\
Leucine 71 & UUA & CUC \\
Leucine 99 & UUG & CUC \\
Leucine 116 & UUG & CUC \\
Leucine 167 & UUG & CUC \\
Leucine 185 & UUG & CUC \\
Leucine 234 & UUG & CUC \\
Leucine 280 & UUG & CUC \\
Leucine 313 & UUG & CUC \\
\hline
\end{tabular}




\section{The translation of iraP requires the $\mathrm{ms}^{2} \mathrm{i}^{6} \mathrm{~A} 37$ tRNA modification for decoding of MiaA-sensitive leucine codons}

To determine if the $\mathrm{ms}^{2} \mathrm{i}^{6} \mathrm{~A} 37$ modification was required for optimal translation of other HULC proteins, we examined the leucine codon usage of several arbitrarily selected genes related to rpoS regulation. In addition, we acquired a table documenting the leucine codon usage of every ORF in the E. coli genome (Supplemental Table S7). We decided to test our hypothesis on iraP, which encodes an anti-adaptor that plays a role in rpoS stability (Bougdour et al. 2006). iraP is a HULC protein, with a UUX-Leucine codon usage ratio of 0.46 , which is twofold greater than the expected UUXLeucine codon ratio and higher than the UUX-Leucine codon usage ratio for rpoS of 0.29 (Table 3). The secondary rationales for this selection were the size of iraP and its involvement in the rpoS regulatory circuitry. The very short ORF of iraP, 258 nucleotides, was ideal for rapid and efficient leu codon engineering. Finally, we reasoned that linking another HULC protein with $\mathrm{ms}^{2} \mathrm{i}^{6} \mathrm{~A} 37$ sensitivity would increase the possibility of identifying a physiological relationship between $\mathrm{ms}^{2} \mathrm{i}^{6} \mathrm{~A} 37$ and rpoS expression.

We constructed an arabinose-inducible iraP258-lacZ translational fusion and a parallel version in which the six UUG and UUA le $u$ codons were changed to CUU codons $\left[\mathrm{P}_{B A D^{-}}\right.$iraP258(leu*3)lac $Z$ ]. The $\mathrm{P}_{B A D}$-iraP258-lac $Z$ translation fusions contain the entire iraP ORF except for the stop codon (Fig. 1D). At 15 min, following arabinose induction, the $\mathrm{miaA}^{+} / \mathrm{miaA}^{-}$ratio for the $\mathrm{P}_{B A D^{-}}$ iraP258-lacZ fusions is $15: 1$, and the ratio decreases with time but is still two- to threefold decreased after $30 \mathrm{~min}$ (Fig. 1C; Supplemental Table S5). This suggests that MiaA ( $i^{6} \mathrm{~A} 37$ tRNA modification) is necessary for efficient iraP translation. In contrast, the $\mathrm{P}_{B A D^{-}}$ iraP258(leu*3)-lacZ was only slightly affected by the absence of MiaA (Fig. 1C). The similar $\beta$-galactosidase-specific activity of the miaA ${ }^{+}$and miaA $^{-} \mathrm{P}_{B A D^{-}}$ iraP258(leu*3)-lac Z translational fusions suggests that iraP UUX leu to CXX leu codon mutations suppress the MiaA (i $\left.{ }^{6} \mathrm{~A} 37\right)$ requirement for iraP translation (Fig. 1C; Supplemental Table S5). Overall these results suggest that UUX leu codons are needed for optimal translation of iraP and changing UUX leu to CUX leu codon mutations decreases the efficiency of iraP translation in the wild-type $\left(\mathrm{miaA}^{+}\right)$strain.

\section{Overexpression of leuX (tRNA $\mathrm{CAA}$ ) suppresses the MiaA requirement for rpoS, but not for rpoS $\left(\mathrm{Ieu}^{*} 3\right)$ or iraP translation}

We hypothesized that overexpression of leucine tRNAs may suppress the effect of the absence of the $i^{6} \mathrm{~A} 37$ tRNA modification on rpoS translation. If the tRNAs that read UUX leu codons are limiting, leading to the need for the modification for more efficient use, overexpression of these leucine tRNAs may suppress the MiaA requirement for rpoS translation. To test this hypothesis, we measured induction of $\mathrm{P}_{B A D^{-}}$rpoS990-lacZ, in the absence of MiaA, but with overexpression of tRNAs that recognize UUXLeu codons (Fig. 2). Plasmids carrying either leuX (CAA anticodon) or leuZ (UAA anticodon), under control of an IPTG-inducible promoter, were transformed into $r s s B^{-}$ $\mathrm{miaA}^{+}$and $r s s B^{-}$miaA $^{-} \mathrm{P}_{B A D^{-}}$rpoS990-lacZ translational fusion strains. The activities of these strains were measured at 5-min intervals after addition of arabinose (Fig. 2A,C), or arabinose and IPTG (to induce the tRNAs) (Fig. 2B,D). The leuX-encoding plasmid had very little effect on a wild-type $\left(\mathrm{miaA}^{+}\right)$strain, with or without IPTG induction (Fig. 2A,B). However, the leuX-encoding plasmid
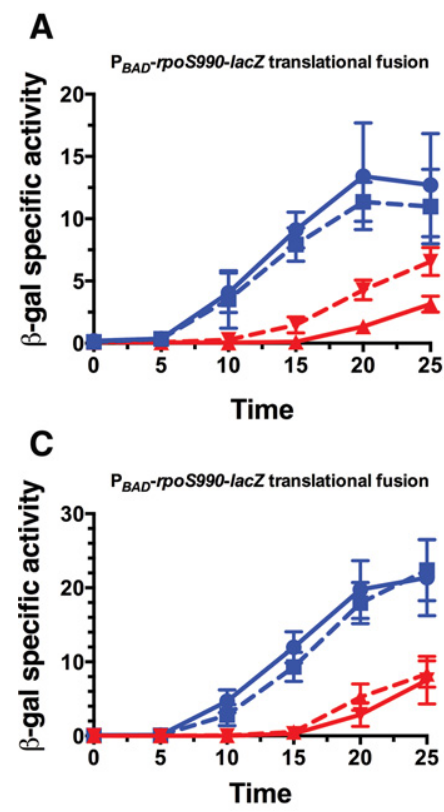

B

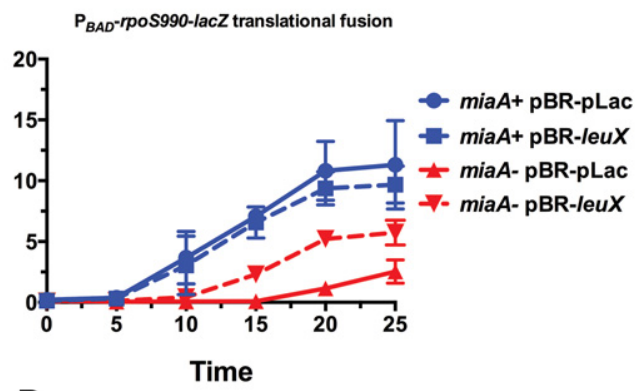

D

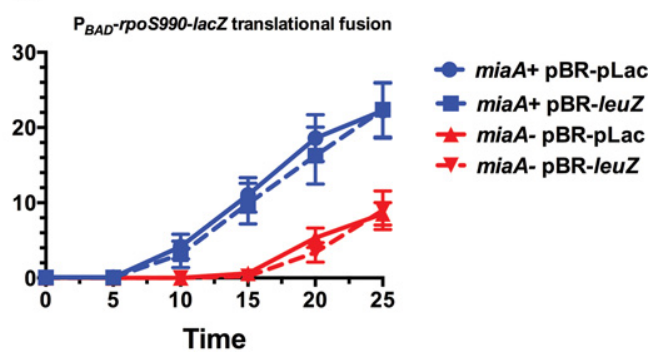

FIGURE 2. The effect of tRNA ${ }^{\text {leuX }}$ and tRNA ${ }^{\text {leuZ }}$ expression on wild-type and miaA $\mathrm{P}_{B A D^{-}}$ rpoS990-lacZ translational fusion activity. The $r s s B^{-} \mathrm{miaA}^{+}$(KMT30003) and $r s s B^{-} \mathrm{miaA}^{-}$ (KMT30011A) $\mathrm{P}_{B A D^{-}}$rpoS990-lacZ translational fusion strains containing plasmids pBR-pLac (KMT30029 and KMT30035, respectively) or pBR-pLac-leuX (KMT30030 and KMT30036) were assayed for $\beta$-galactosidase-specific activity following arabinose induction without $(A)$ or with $(B)$ IPTG. Each value represents the mean of at least three replicate experiments; the error bars represent the standard error of the mean (SEM). The $r s s B^{-}$miaA $A^{+}$(KMT30003) and $r s s B^{-}$ miaA $^{-}$(KMT30011A) $\mathrm{P}_{B A D^{-}}$rpoS990-lacZ translational fusion strains containing plasmids pBRpLac (KMT30029 and KMT30035, respectively) or pBR-pLac-leuZ (KMT30031 and KMT30037) were assayed for $\beta$-galactosidase-specific activity following arabinose induction without $(C)$ or with $(D)$ IPTG. Each value represents the mean of at least three replicate experiments; the error bars represent the standard error of the mean (SEM). 
significantly increased expression of the fusion in the miaA mutant strain (Fig. 2A,B). The increase in expression in the absence of IPTG suggests that there is leaky expression of the tRNA ${ }_{\text {CAA }}^{\text {LeuX }}$. No increase in the $\beta$-galactosidase-specific activity of the miaA ${ }^{-} \mathrm{P}_{B A D^{-}}$-rpoS990-lacZ translational fusion was observed in a parallel experiment in which leuZ, encoding tRNA ${ }_{\text {UAA }}^{\text {LeuZ }}$ was overexpressed (Fig. 2C,D). Taken together, this suggests that overexpression of tRNA ${ }^{\text {LeuX }}{ }_{\text {CAA }}$, but not tRNA ${ }_{\text {UeuA }}^{\text {UAA }}$, can partially suppress the MiaA requirement for rpoS translation.

We hypothesized that LeuX suppression of the $\mathrm{ms}^{2} \mathrm{i}^{6} \mathrm{~A} 37$ requirement for rpoS translation would not be observed when the MiaA-sensitive leucine codons were removed. To test this hypothesis, we transformed pBR-pLac and pBRpLac-leuX into $r s s B^{-}$miaA $^{+}$and $r s s B^{-}$miaA $A^{-}$genetic backgrounds of this $\mathrm{P}_{B A D^{-}} r p o S 990($ leu*3)-lacZ translational fusion and measured the $\beta$-galactosidase-specific activity of the resulting strains after arabinose induction (Fig. 3A). We observed no effect of tRNA ${ }_{\text {LeuX }}{ }_{\text {CAA }}$ expression on the $\beta$-galactosidase-specific activity of the fusion in the presence or absence of MiaA. This suggests that the tRNA ${ }^{\text {LeuX }}{ }_{\text {CAA }}$ suppression of the MiaA requirement for rpoS translation is

A
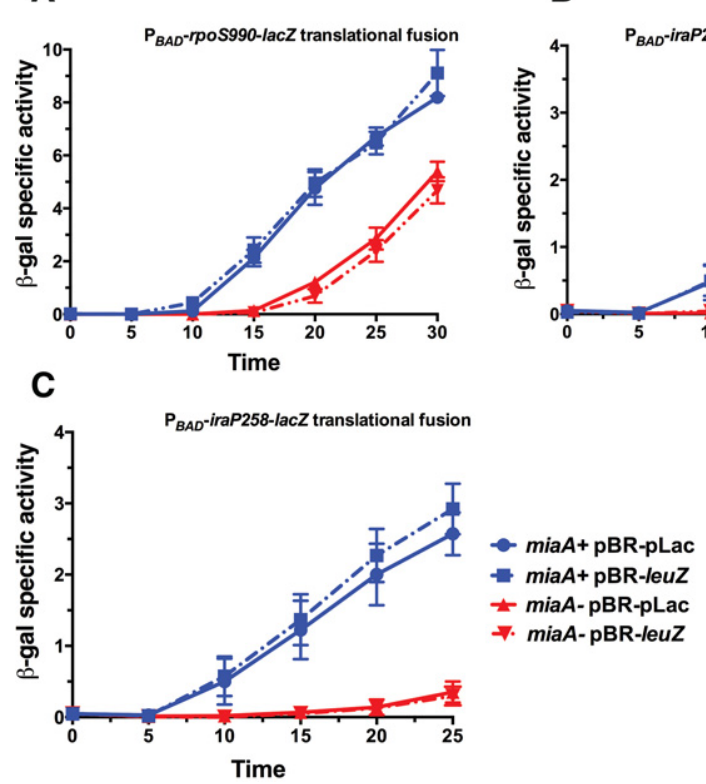

FIGURE 3. The tRNA ${ }^{\text {leuX }}$ suppression assay for the miaA effect on $r p o S\left(l e u^{*} 3\right)$ and iraP. $(A)$ The $r_{s s B^{-}}$miaA $^{+}$(KMT33001) and $r s s B^{-}$miaA $^{-}$(KMT33002) $\mathrm{P}_{B A D^{-}}$(KpoS990(leu*3)-lacZ translational fusion strains containing plasmids pBR-pLac (KMT30029 and KMT30035, respectively) or pBR-pLac-leuX (KMT30030 and KMT30036) were assayed for $\beta$-galactosidase-specific activity following arabinose and IPTG induction. (B) The miaA ${ }^{+}$(KMT45000) and $\mathrm{miaA}^{-}$ (KMT45002) $\mathrm{P}_{B A D}$-iraP258-lacZ translational fusion strains containing plasmids pBR-pLac (KMT45003 and KMT45006, respectively) or pBR-pLac-leuX (KMT45004 and KMT45007) were assayed for $\beta$-galactosidase-specific activity following arabinose and IPTG induction. $(C)$ The miaA $^{+}$(KMT45000) and miaA ${ }^{-}$(KMT45002) $\mathrm{P}_{B A D^{-} \text {-iraP258-lacZ translational fusion strains }}$ containing plasmids pBR-pLac (KMT45003 and KMT45006, respectively) or pBR-pLac-leuZ (KMT45005 and KMT45008) were assayed for $\beta$-galactosidase-specific activity following arabinose and IPTG induction. Each value represents the mean of at least three replicate experiments; the error bars represent the standard error of the mean (SEM). through the decoding of UUA's and UUG's leucine codons in the rpos ORF.

We carried out similar experiments with the $\mathrm{P}_{B A D}$-iraP258lacZ translational fusions (Fig. 3B,C). Neither tRNA ${ }_{\text {CAA }}^{\text {LeuX }}$ nor tRNA ${ }_{\text {UAA }}^{\text {LeuZ }}$ had a significant effect on the $\beta$-galactosidase-specific activity of the $\mathrm{P}_{B A D}$-iraP258-lacZ translational fusion in the absence of MiaA (Fig. 3B,C). This suggests that tRNA ${ }_{\text {CAA }}^{\text {LeuX }}$ is not or is only a minimally effective suppressor of the MiaA requirement for iraP translation. Possible explanations for the difference between $r p o S$ and iraP include the higher number of UUX leu codons in rpoS (13) compared to iraP (six) or the existence of tandem UXX-leu codons in iraP versus the absence of such in rpoS (Tables 1,2).

\section{Mutation of UUA-Leu to CUU-Leu or CUC-Leu codons suppress the MiaA requirement for}

To further characterize the role of the $\mathrm{ms}^{2} \mathrm{i}^{6} \mathrm{~A} 37$ tRNA modification in the decoding of Leu codons in rpoS and iraP, we constructed translational lac $Z$ fusions to alleles of rpoS and iraP where either UUA- or UUG-Leucine codons were removed and replaced with either a CUC or CUU leucine codon. We designate the UUA-Leu to CUU-Leu or CUC-Leu rpoS alleles as $l e u^{*} 1$ alleles. We designate the UUG-Leu to CUC-Leu rpoS alleles as $l e u^{*} 2$ alleles. The precise amino acid number and mutation are listed in Table 1 . We then tested the $\beta$-galactosidase-specific activity of these fusions as described in the Materials and Methods section. We also repeated the assay in Figure 1A, with wild-type and $l e u^{*} 3$ rpoS alleles, as a control for the $l e u^{*} 1$ and $l e u^{*} 2$ rpoS alleles (Fig. 4A). In the presence of MiaA, the rpoS $l e u^{*} 1$ allele has a modest decrease in $\beta$-galactosidase-specific activity between 10 and $25 \mathrm{~min}$ following induction (Fig. 4B). The $\mathrm{miaA}^{+}$ratio of wild type to $l e u^{*} 1$ is increased by 1.2 to 1.5 -fold at or beyond $15 \mathrm{~min}$ of induction (Supplemental Table S4). This suggests that the UUA-Leu to CXX-Leu silent codon mutations slightly decrease efficiency of rpoS translation. In the absence of $\mathrm{MiaA}$, at or beyond $15 \mathrm{~min}$ of induction, the $\beta$-galactosidase-specific activity of the $\mathrm{P}_{B A D^{-}}$-rpoS990(leu*1)-lacZ allele is at least twofold greater than the $\mathrm{P}_{B A D^{-}} r p o S 990$ lac $Z$ allele. The activities of the $\mathrm{miaA}^{+}$ and miaA $^{-} \mathrm{P}_{B A D^{-}}$rpoS990(leu*1)-lacZ translational fusion are relatively similar in comparison to the $\mathrm{miaA}^{+}$and $\mathrm{miaA}^{-}$ $\mathrm{P}_{B A D^{-}}$rpoS990-lacZ translational fusion 
TABLE 2. Silent leucine mutations in the iraP leu* alleles

\begin{tabular}{lcc}
\hline Amino acid & Original codon & Leu* recoded codon \\
\hline Leucine 8 & UUG & CUU \\
Leucine 9 & UUA & CUU \\
Leucine 29 & UUG & CUU \\
Leucine 48 & UUG & CUU \\
Leucine 80 & UUA & CUU \\
Leucine 81 & UUG & CUU \\
\hline
\end{tabular}

(Fig. 4B). The $\mathrm{miaA}^{+} / \mathrm{miaA}^{-}$ratio of the $\mathrm{P}_{B A D^{-}}$rpoS990 (leu*1)-lacZ and $\mathrm{P}_{B A D^{-}}$-rpoS990-lacZ translational fusions, 15 min following induction, are 1.8 and 23 , respectively. This ratio is much closer to 1.0-fold increase in the $l e u^{*} 1$ fusion as opposed to the wild-type fusion (Supplemental Table S3). This suggests that MiaA and the $\mathrm{ms}^{2} \mathrm{i}^{6} \mathrm{~A} 37$ tRNA modification are necessary for efficient translation of UUA-Leucine codons within the rpoS ORF.

\section{UUG-Leu to CXX-Leu codon mutations decrease rpoS translational efficiency}

In the presence of MiaA, the leu* 2 alleles are severely defective for translational $\beta$-galactosidase-specific activity, decreased by approximately fourfold starting at $10 \mathrm{~min}$ after induction (Fig. 4C; Supplemental Table S4). This suggests that, independent of MiaA and the $\mathrm{ms}^{2} \mathrm{i}^{6} \mathrm{~A} 37$ tRNA modification, the presence of UUG-Leucine codons is critical for efficient translation of the rpoS ORF. Furthermore, the $\beta$ galactosidase-specific activity of the miaA ${ }^{+} \mathrm{P}_{B A D^{-}} r p o S 990$ (leu*2)-lac $Z$ translational fusion is nearly identical to the miaA $^{-} \mathrm{P}_{B A D^{-}}$rpoS990(leu*2)-lacZ translational fusion (Fig. 4; Supplemental Table S3). However, the severely compromised rpoS translation seen in the absence of UUG possibly makes this explanation too simple. Interestingly, there is no statistically significant difference, at the 30 -min time point following induction, between $\mathrm{miaA}^{+}$and $\mathrm{miaA}^{-}$activities of the $l e u^{*} 1$ and $l e u^{*} 2$ alleles of the $\mathrm{P}_{B A D^{-}}$rpoS990-lacZ translational fusion (Fig. 4D). In contrast, there is a statistically significant difference, at the 30-min time point following induction, between $\mathrm{miaA}^{+}$and $\mathrm{miaA}^{-}$activities of the wildtype and $l e u^{*} 3$ alleles of the $\mathrm{P}_{B A D^{-}}$rpoS990-lacZ translational fusion (Fig. 4D). The removal of UUA-Leu only or UUG-Leu codons only, as opposed to the removal of both, results in stronger suppression of the MiaA requirement during rpoS expression. The reason for this is unclear.

\section{UUA-Leu to CUU-Leu or UUG-Leu to CUU-Leu codon mutations modulate iraP translational efficiency and partially suppress the MiaA requirement during iraP translation}

While it is clear from data in Figure 1C that removal of both UUA-Leucine and UUG leu codons within iraP suppresses the MiaA effect during iraP translation, we sought to further characterize and define the relative contributions of UUA leu and UUG leu to the MiaA requirement for iraP translation just as we did for $r p o S$ translation. We created two additional alleles of the $\mathrm{P}_{B A D^{-}}$iraP258-lac $Z$ translational fusion, $\mathrm{P}_{B A D^{-}}$ iraP258(leu*1)-lac $Z$ and $\mathrm{P}_{B A D^{-}}$-iraP258(leu*2)-lac $Z$. $\mathrm{P}_{B A D^{-}}$ iraP258(leu*1)-lacZ consists of UUA-Leucine to CUULeucine codon changes alone (Table 2). $\mathrm{P}_{B A D}$-iraP258 (leu*2)-lacZ consists of UUG-Leucine to CUU-Leucine codon changes alone (Table 2). We measured the $\beta$-galactosidase-specific activity of these fusions as described in the Experimental Design section of Materials and Methods. Upon comparison of the miaA ${ }^{+} \mathrm{P}_{B A D^{-}}$iraP258(leu*1)-lacZ and miaA ${ }^{+} \mathrm{P}_{B A D^{-}}$-iraP258-lac $Z$ translational fusion $\beta$-galactosidase-specific activity after induction, it is clear that the leu* 1 mutation increases iraP translational efficiency (Fig. 5B), most evident at 15-30 min following induction (Fig. 5B). The miaA ${ }^{+} \mathrm{P}_{B A D^{-} \text {-iraP258-lacZ/ }} \mathrm{P}_{B A D^{-} \text {-iraP258(leu*1)-lacZ ra- }}$ tio is between -1.6 to -1.7 during this time period, demonstrating an increase in iraP translational efficiency in the absence of UUA-Leucine codons (Supplemental Table S6). The $\beta$-galactosidase-specific activity of the miaA ${ }^{-} \mathrm{P}_{B A D^{-}}$ iraP258(leu*1)-lacZ fusion is 2.1-fold less than the miaA ${ }^{+}$

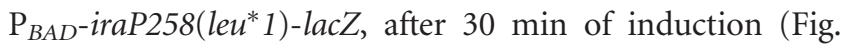
5B; Supplemental Table S5). This is slightly smaller than the 2.8-fold decrease seen after 30 min (Supplemental Table S5). The miaA $^{+} /$miaA $^{-} \beta$-galactosidase-specific activity ratio, 15 min after induction, was 1.8 in the $l e u^{*} 1$ allele versus 15 in the wild-type (non leu*) allele of the $\mathrm{P}_{B A D^{-}}$-iraP258-lac $Z$ translational fusion (Supplemental Table S5). This suggests that the UUA-Leucine to CUU-Leucine codon changes lead to partial suppression of the MiaA requirement for iraP translation.

We measured the $\beta$-galactosidase-specific activity of the $\mathrm{P}_{B A D^{-} \text {-iraP258(leu*2)-lac } Z \text { translational fusion after induc- }}$ tion, in the presence and absence of MiaA to determine the relative contribution of UUG-Leucine codons to iraP translation (Fig. 5; Supplemental Table S6). UUG-Leucine to CUU-

\begin{tabular}{lcll}
\multicolumn{3}{l}{ TABLE 3. Leucine codon usage in $r p o S$ and iraP } \\
\hline & & \multicolumn{2}{c}{ Actual ratio } \\
\cline { 3 - 4 } Leucine codon & Expected ratio & rpoS & iraP \\
\hline UUA & 0.11 & $0.09^{\mathrm{a}}$ & $0.15^{\mathrm{b}}$ \\
UUG & 0.11 & $0.20^{\mathrm{a}}$ & $0.31^{\mathrm{b}}$ \\
CUU & 0.10 & 0.11 & 0.15 \\
CUC & 0.10 & 0.02 & 0.08 \\
CUA & 0.03 & 0.00 & 0.00 \\
CUG & 0.55 & 0.57 & 0.31 \\
Total & 1 & 1 & 1
\end{tabular}

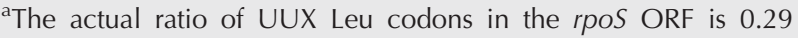
versus an expected UUX codon usage of 0.22 .

${ }^{b}$ The actual ratio of UUX Leu codons in the iraP ORF is 0.46 versus an expected UUX codon usage of 0.22 . 

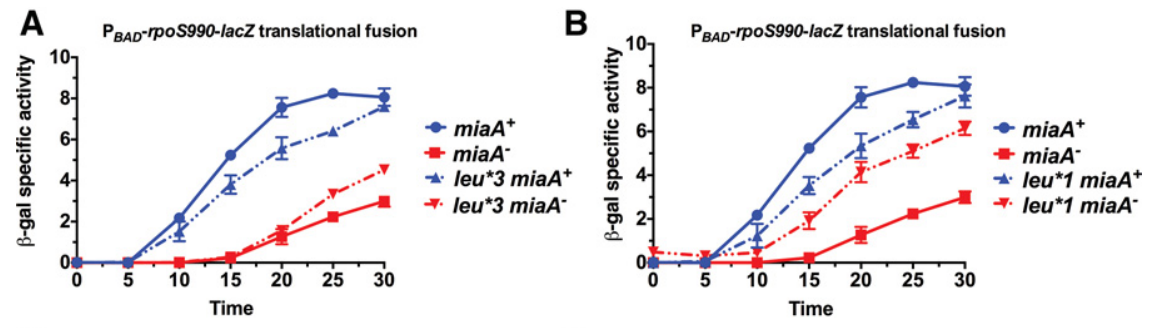

C
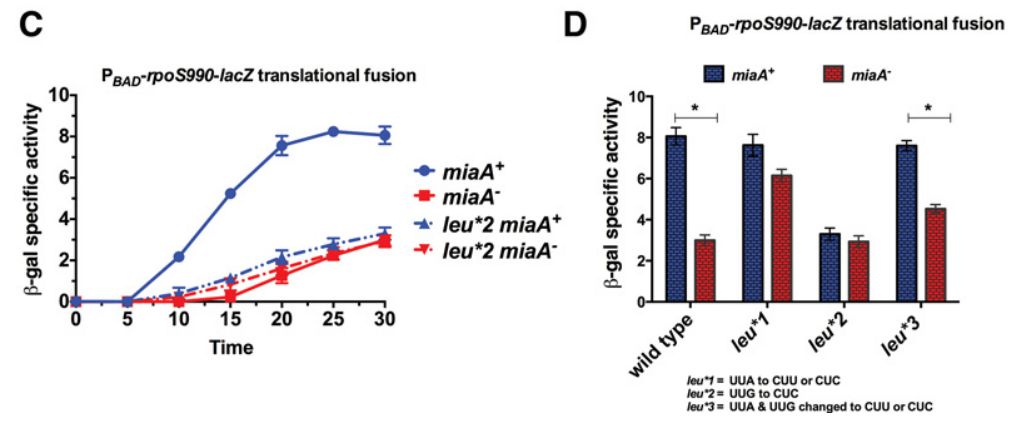

FIGURE 4. Translation of $r p o S$ with UUG- or UUA-Leucine codons replaced with CXX-Leucine codons. (A) The $r s s B^{-}$miaA $^{+}$(KMT33001) and rssB $B^{-}$miaA $^{-}$(KMT33002) $\mathrm{P}_{B A D^{-}}$rpoS990(leu*3)lacZ as well as $r s s B^{-}$miaA $^{+}$(KMT30003) and $r s s B^{-}$mia $A^{-}$(KMT30011A) $\mathrm{P}_{B A D^{-}}$-rpoS990-lacZ translational fusion strains were assayed for $\beta$-galactosidase-specific activity following arabinose induction. (B) The $r s s B^{-}$miaA $^{+}$(KMT) and $r s s B^{-}$miaA $^{-}$(KMT) $\mathrm{P}_{B A D^{-}} r$ poS990(leu*2)-lacZ as well as $r s s B^{-}$miaA $^{+}\left(\mathrm{KMT}^{2} 0003\right)$ and $r s s B^{-}$miaA $^{-}$(KMT30011A) $\mathrm{P}_{B A D^{-}}$rpoS990-lacZ translational fusion strains were assayed for $\beta$-galactosidase-specific activity following arabinose induction. (C) The rssB $B^{-}$miaA $^{+}$(KMT37002) and rssB ${ }^{-}$miaA $A^{-}$(KMT37003) $\mathrm{P}_{B A D^{-}}$rpoS990(leu*2)lacZ as well as $r s s B^{-}$miaA $^{+}$(KMT30003) and $r s s B^{-}$miaA $^{-}$(KMT30011A) $\mathrm{P}_{B A D^{-}}$(KpoS990-lacZ translational fusion strains were assayed for $\beta$-galactosidase-specific activity following arabinose induction. $(D)$ The $r s s B^{-}$miaA $^{+}$(KMT36002) and $r s s B^{-}$miaA $A^{-}$(KMT36003) $\mathrm{P}_{B A D^{-}}$rpoS990 (leu*1)-lacZ, rssB $B^{-}$miaA $^{+}\left(\mathrm{KMT}^{*} 7002\right)$ and rssB $B^{-}$miaA $^{-}$(KMT37003) $\mathrm{P}_{B A D^{-}}$rpoS990(leu*2)lacZ, $r s s B^{-}$miaA $^{+}\left(\mathrm{KMT}_{33001)}\right.$ and $r s s B^{-}$miaA $^{-}$(KMT33002) $\mathrm{P}_{B A D^{-}} r p o S 990($ leu 3$)$-lacZ as well as $r s s B^{-}$miaA $^{+}$(KMT30003) and $r s s B^{-}$miaA $^{-}$(KMT30011A) $\mathrm{P}_{B A D^{-}}$rpoS990-lacZ wildtype translational fusion strains were assayed for $\beta$-galactosidase-specific activity following 30 min of arabinose induction. Each value represents the mean of at least three replicate experiments; the error bars represent the standard error of the mean (SEM). The means between miaA $^{+}$and mia $^{-}$strains in section $D$ were analyzed for statistical significance using $t$-test and the HolmSidak method with $P$-values $<0.05$.

Leucine codons had no effect on the translation of iraP in a miaA $^{+}$host (Fig. 5C; Supplemental Table S6). However, the $\beta$-galactosidase-specific activity of the miaA ${ }^{-} \mathrm{P}_{B A D^{-}}$ iraP258(leu*2)-lac $Z$ translational fusion is higher than the

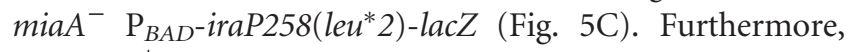
the $\mathrm{miaA}^{+} / \mathrm{miaA}^{-}$ratios in the $l e u^{*} 2$ allele of the $i r a P$ fusion are less than the $\mathrm{miaA}^{+} / \mathrm{miaA}^{-}$ratios in the wild-type allele of the iraP fusion (Fig. 5C,D; Supplemental Table S5). Taken together this suggests that the $l e u^{*} 2$ allele allows for suppression of the MiaA requirement for iraP translation. More precisely, $\mathrm{MiaA}$ is necessary for iraP translation in part due to decoding of UUG-Leucine codons.

\section{MiaB is dispensible for $r p o S$ and iraP translation}

As previously discussed, the MiaA enzyme catalyzes the first step in the synthesis of the $\mathrm{ms}^{2} \mathrm{i}^{6} \mathrm{~A} 37$, the addition of the $\mathrm{i}^{6}$ to the A37 nucleotide (Bartz et al. 1970). The addition of the $\mathrm{ms}^{2}$ group, catalyzed by the MiaB enzyme, requires the presence of the $\mathrm{i}^{6}$ as a prerequisite (Vold et al. 1979).
Although we had previously demonstrated no effect of miaB mutants on rpoS levels (Thompson and Gottesman 2014), we revisited this question using the rpoS and iraP translational fusions. We measured the $\beta$-galactosidase-specific activity of the $\mathrm{P}_{B A D^{-}}$-rpoS990-lac $Z$ and

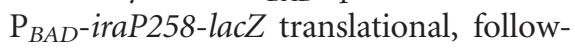
ing arabinose induction (Supplemental Fig. S9). For the rpoS translational fusion, there was a negligible decrease in the $\beta$-galactosidase-specific activity of the fusion in the miaB mutant, at 15-30 min following arabinose induction. For the iraP translational fusion, there was a negligible decrease in the $\beta$-galactosidase-specific activity of the fusion in the miaB mutant, at 20-30 min following arabinose induction. Taken together, this confirms our previous finding, and shows that the $\mathrm{ms}^{2}$ portion of the $m s^{2} i^{6} \mathrm{~A} 37$ tRNA modification is dispensable for full rpoS and iraP translation. The $i^{6} \mathrm{~A} 37$ portion of the $\mathrm{ms}^{2} \mathrm{i}^{6} \mathrm{~A} 37$ tRNA modification is responsible for its role in translation of rpoS and iraP.

\section{DISCUSSION}

\section{Prediction of $i^{6} \mathrm{~A} 37$ modification tunable transcripts (MoTTs) through leucine codon usage}

Endres et al. (2015) define transcripts with codon usage different from that of average transcripts, as modification tunable transcripts (MoTTs). In their model, under normal growth conditions, MoTTs are moderately expressed in the presence of tRNA modifications (Endres et al. 2015). Levels of tRNA modification, and therefore the translation of MoTTs, change during stress or damage conditions (Endres et al. 2015). Our findings for rpos and iraP establish them as MoTTs, adding MiaA modification and UUX codon usage as new examples of this phenomenon.

We hypothesized that UUX-Leucine codon enrichment within an ORF may correlate with MiaA ( $\left.{ }^{6} A 37\right)$ sensitivity in genes other than rpoS (Thompson and Gottesman 2014). The data in this study strongly support the utility of using leucine codon usage ratios to predict the necessity of the $i^{6} \mathrm{~A} 37$ tRNA modification during translation of a given protein and provides a possible method for the identification of this subclass of MoTTs as HULC genes. We observed that the ratio of UUX-Leu codons in iraP is 0.46 , which is even greater than the 0.29 ratio seen in rpos (Table 3). While both ratios are higher than the expected 0.22 , the ratio is 

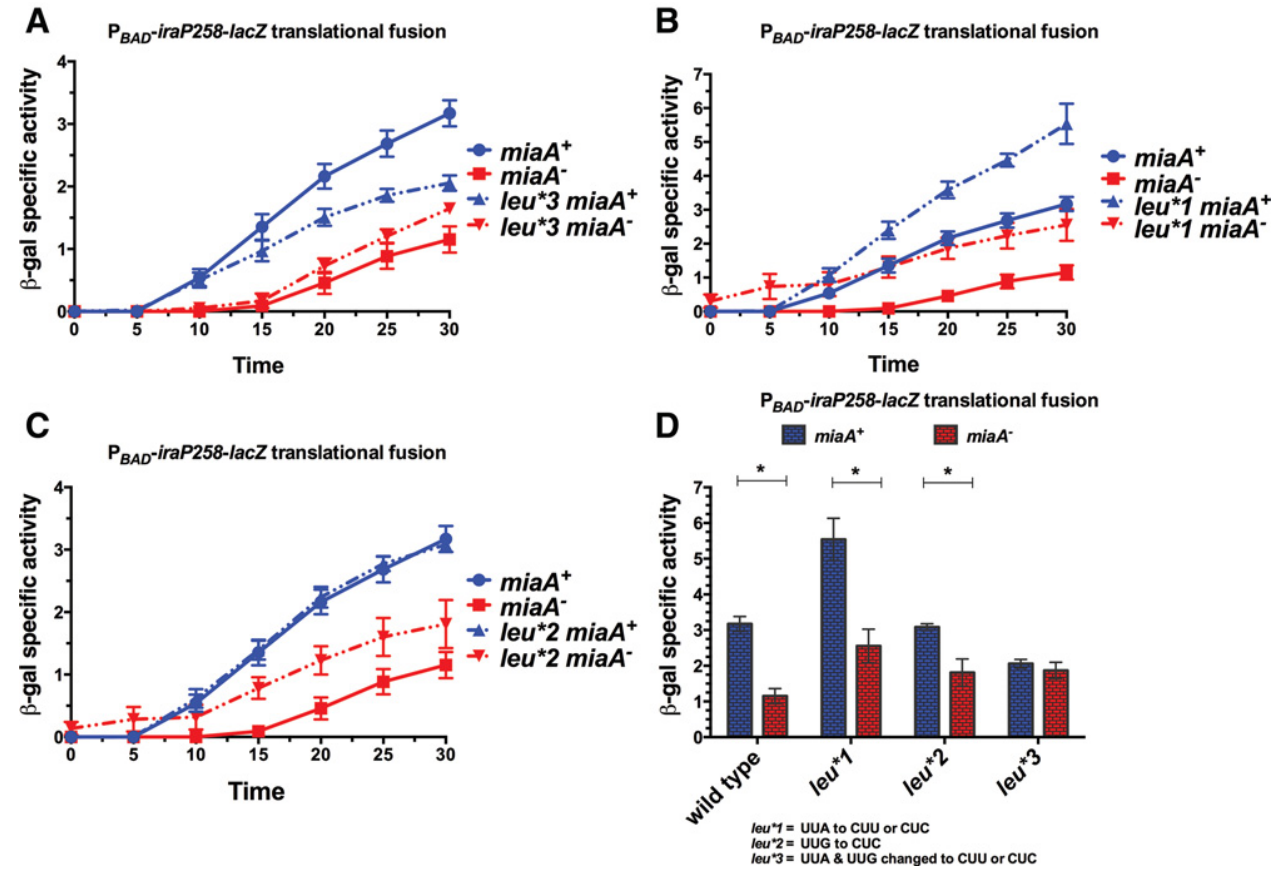

FIGURE 5. Translation of iraP with UUG- or UUA-Leucine codons replaced with CXX-Leucine codons. (A) The $\mathrm{miaA}^{+}\left(\mathrm{KMT}^{2} 5000\right)$ and miaA $^{-}$ (KMT45002) $\mathrm{P}_{B A D^{-}}$iraP258(leu*3)-lacZ as well as miaA ${ }^{+}\left(\mathrm{KMT}^{*}\right.$ 2000) and miaA ${ }^{-}$(KMT42002) $\mathrm{P}_{B A D^{-}}$-258-lacZ translational fusion strains were as-

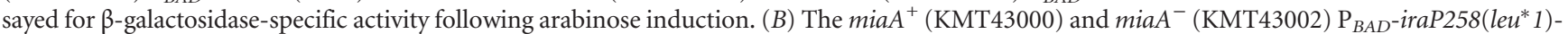

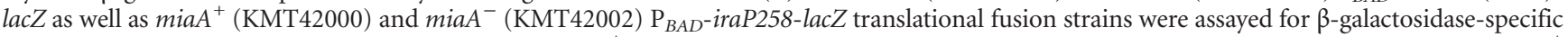
activity following arabinose induction. (C) The $\mathrm{miaA}^{+}\left(\mathrm{KMT44000)}\right.$ and $\mathrm{miaA}^{-}\left(\mathrm{KMT44002)} \mathrm{P}_{B A D^{-} \text {iraP258(leu }}{ }^{*}\right.$ )-lacZ as well as miaA ${ }^{+}$

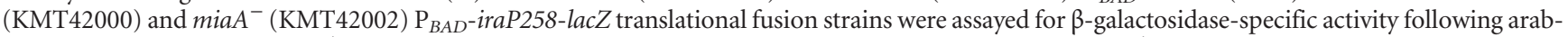
inose induction. (D) The miaA ${ }^{+}$(KMT43000) and miaA ${ }^{-}$(KMT43002) $\mathrm{P}_{B A D^{-} \text {-iraP258(leu*1)-lacZ, the miaA }}{ }^{+}$(KMT44000) and miaA ${ }^{-}$(KMT44002)

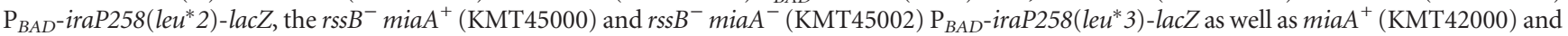

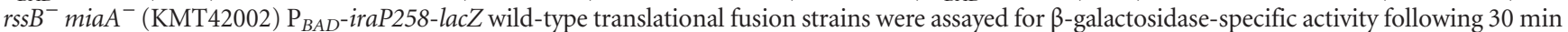
of arabinose induction. Each value represents the mean of at least three replicate experiments; the error bars represent the standard error of the mean (SEM). The means between $\mathrm{miaA}^{+}$and $\mathrm{miaA}^{-}$strains in section $D$ were analyzed for statistical significance using $t$-test and the Holm-Sidak method with $P$-values $<0.05$.

much higher in iraP. Further bioinformatics searches, along with biochemical and genetic analysis, will be needed to further evaluate the predictive power associated with the correlation of UUX-Leu codon usage and sensitivity to the lack of MiaA for putative HULC proteins. These investigations are likely to yield more insight into the regulatory nature of the $i^{6} \mathrm{~A} 37$ tRNA modification in $E$. coli and other biological systems.

\section{UUG-Leucine and UUA-Leucine codon usage in rpoS and iraP}

There are differences in how UUA and UUG codons are used in $r p o S$ and $i r a P$, in relation to the presence of the $i^{6} \mathrm{~A} 37$ modification. Our $l e u^{*}$ mutations were created with the idea that UUX-Leucine to CUX-Leucine mutations would suppress the MiaA requirement for full rpoS translation. The leu*3 mutation suppressed the MiaA effect in both rpoS and iraP (Figs. 4, 5). However, the suppression was more pronounced in iraP, as miaA $A^{+}$and miaA $^{-}$levels were identical (Fig. 5). The reason for this is unclear, particularly since iraP has seven fewer UUX-Leu codons than rpoS. Both, $l e u^{*} 1$ and $l e u^{*} 2$ mutations, representing the UUA-CUX and UUG-CUX substitution mutations, respectively, demonstrated stronger MiaA suppression than the $l e u^{*} 3$ mutation for reasons that are not clear. However, this suggests that UUA-Leu and UUG-Leu substitutions, individually, are more effective at suppressing the MiaA effect than UUA and UUG substitutions collectively. It should be noted that the rpoS transcript is approximately three times longer than the iraP transcript. UUG-Leucine codons are more frequent within the rpoS and iraP transcripts than UUA-Leucine codons. The number of UUX codons in iraP and rpoS transcripts is seven and 12, respectively. There are two instances within the iraP transcript where UUX codons occur in tandem, once at leucine 8 and leucine 9 and again at leucine 80 and leucine 81. MiaA affects the translation of the 14 amino acid $\operatorname{trp}$ leader region, $\operatorname{trp} L$, by influencing the translation rate of two tandem Trp codons (Trp 10 and Trp11) by $\mathrm{ms}^{2} \mathrm{i}^{6} \mathrm{~A} 37$-modified tRNA ${ }^{\text {Trp }}$ (Landick et al. 1990). This tandem duplication of UUX-Leucine codons is not seen in the rpoS transcript. These two independent tandem duplications of UUX-Leucine codons in the iraP ORF likely contribute significantly to MiaA sensitivity during iraP translation. Undermodified tRNA $^{\text {Leu }}$ may 
contribute to ribosome pausing or stalling at each of these two tandem duplications contributing to decreased translational speed and accuracy.

\section{The steady-state levels of rpoS have an additional level of regulatory fine-tuning}

rpoS is subject to regulation at multiple levels, including the translation of the rpoS ORF, through SsrA, MiaA, AceE, and the presence of rare codons (Ranquet and Gottesman 2007; Kolmsee and Hengge 2011; Thompson and Gottesman 2014; Battesti et al. 2015). While there have been extensive studies on the regulation of the synthesis and activity of tRNA modification enzymes, the precise cellular or environmental signals that influence the post-transcriptional levels and post-synthesis activities of many tRNA modifications are elusive (Winkler 1998). This is also true of the $i^{6} \mathrm{~A} 37$ modification and the activity of MiaA in E. coli. The leucine codon usage relationship suggests that leucine availability may provide a physiological condition whereby the $i^{6} \mathrm{~A} 37$ modification is most critical for expression of $r p o S$ synthesis. Lrp, the leucine-responsive regulatory protein, is a global regulatory protein whose regulatory action is partially dependent upon leucine (Ernsting et al. 1992; Lin et al. 1992; Platko and Calvo 1993). Mutations in $\operatorname{lrp}$, as well as rpoS, confer a growth advantage during stationary phase, or GASP, phenotype (Zambrano et al. 1993; Zinser and Kolter 2000). In addition, the transcription factor LeuO regulates DsrA, one of the small RNAs that stimulate rpoS translation (Klauck et al. 1997; Repoila and Gottesman 2001). LeuO also regulates the leucine biosynthesis operon, leuABCD (Chen et al. 2001; Stratmann et al. 2012). These observations provide clues into a stationary phase network that connects leucine metabolism and rpoS synthesis.

There are several environmental signals that feed into the rpoS synthesis pathway through the small regulatory RNAs that stimulate its translation and anti-adaptors that promote its protein stability. Low temperature (DsrA), cell surface stress (RprA), aerobiosis (ArcZ), phosphate starvation (iraP), magnesium starvation (IraM), and DNA Damage (IraD) all act on RpoS synthesis or stability (Sledjeski et al. 1996; Majdalani et al. 2002; Bougdour et al. 2006, 2008; Mandin and Gottesman 2010). It would be useful to consider the possibility, and investigate, whether $\mathrm{ms}^{2} \mathrm{i}^{6} \mathrm{~A} 37$ modification levels are modulated under these conditions that are known to stimulate Rpos levels. This may lead to greater insight into the physiological and regulatory nature of the $\mathrm{ms}^{2} \mathrm{i}^{6} \mathrm{~A} 37$ tRNA modification.

MiaA is encoded directly upstream of and co-transcribed with $\mathrm{Hfa}$, which is necessary for rpoS translation mainly through acting as a chaperone for the activating sRNAs (Tsui and Winkler 1994; Tsui et al. 1994). This tandem coding of MiaA and Hfq is highly conserved throughout the prokaryotic domain. This and our previous work offer one possible explanation, the regulation of rpoS translation, for this conserved synteny of miaA and $h f q$. It is likely that further bioinformatics and experimental analysis will uncover other mRNAs whose translation is regulated by both Hfq (possibly through Hfq-dependent sRNAs) as well as the $i^{6} A 37$ tRNA modification.

There is some residual $\beta$-galactosidase-specific activity of the rpoS and iraP leu* fusions, in which all UUX leu codons have been changed to CUX codons, in the absence of miaA (Figs. 1, 4, 5). This suggests that MiaA may be necessary for the decoding of other (non-leu) codons within rpoS and iraP, or have some other indirect effects on rpoS and iraP translation. We previously identified $t s a E$ as a gene that now falls into the HULC designation of proteins and consequently may be an $i^{6} \mathrm{~A} 37$ MoTT (Thompson and Gottesman 2014). The TsaE protein is also encoded upstream of miaA and $h f q$ (Tsui and Winkler 1994). The TsaE protein, along with $\mathrm{TsaC}, \mathrm{TsaD}$, and $\mathrm{TsaB}$, form an enzyme that catalyzes the addition of the $N^{6}$-L-threonylcarbamoyladenine ${ }^{37}$ $\left(t^{6}\right.$ A37) modification on ANN decoding tRNAs (Deutsch et al. 2012; Zhang et al. 2015). It is possible that translation of both rpoS and iraP are sensitive to loss of the $\mathrm{t}^{6} \mathrm{~A} 37$ modification, and that $i^{6} \mathrm{~A} 37$ deficiency affects $r p o S$ and iraP levels in part indirectly by limiting TsaE translation. We are currently investigating the possible role of $t^{6} \mathrm{~A}$ on $r p o S$ and iraP levels to test this hypothesis.

\section{Implications for comparative functional genomics of tRNA isopentenyl transferases}

We previously noted other proteins within the prokaryotic domain that are sensitive to MiaA levels (Thompson and Gottesman 2014). These proteins include Agrobacterium tumefaciens vir and Shigella flexneri VirF (Gray et al. 1992; Durand et al. 1997, 2000). In addition, Streptomyces coelicolor bld mutant phenotype is suppressed by overexpression of

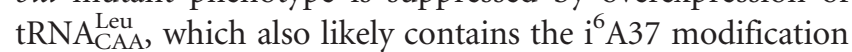
(Pettersson and Kirsebom 2011). The UUX-Leucine codon usage in Shigella flexneri VirF is indicative of MiaA sensitivity (Thompson and Gottesman 2014). Based on our observations in E. coli K12, it is likely that experimental analysis of leucine codon usage within these genes will yield insights into how UUX-Leu codons influence the translation of MiaA-sensitive proteins in other bacterial species.

The MiaA gene is also highly conserved among other biological domains, with homologs in yeast and humans, MOD5 and TRIT1, respectively (Martin and Hopper 1982; Golovko et al. 2000; Lamichhane et al. 2013b; Yarham et al. 2014). TRIT1 is particularly interesting due to its role as a putative lung adenocarcinoma tumor suppressor, as its expression was significantly down regulated in lung adenocarcinomas (Spinola et al. 2005). A genome-wide association study of a single nucleotide polymorphism (SNP) mapping to TRIT1, in different ethnic populations, demonstrated statistically significant links between a TRIT1 Phe202Leu genotype and lung cancer survival rates (Spinola et al. 2007). This suggests that 
an association of leucine codon usage and tRNA isopentenyl transferase activity may be highly conserved well beyond the bacterial domain.

\section{Expansion of the rpoS regulatory network in $E$. coli}

Prior to this work, the mechanism by which MiaA exerted its effect on rpoS expression was undefined. Our data strongly suggest that the MiaA requirement for $r p o S$ expression is partially through promotion of translational efficiency at MiaAsensitive UUA- and UUG-Leucine codons in the rpoS ORF. The rpoS ORF is somewhat enriched for these leucine codons in comparison to other proteins that are gene regulators or members of the transcriptional regulatory machinery (Thompson and Gottesman 2014). Our current model for the role of MiaA in rpoS regulation is illustrated in Figure 6 . In the presence of active, or excess MiaA, $i^{6} \mathrm{~A} 37$-modified tRNA ${ }^{\text {Leu }}$ CAA efficiently recognizes UUA or UUG codons within the rpoS and iraP ORFs, leading to optimal translation of $r p o S$ and iraP ORFs (Fig. 6). iraP levels contribute to rpoS stability, further increasing steady-state levels of rpoS (Fig. 6). In the absence of MiaA, or the presence of inactive MiaA, unmodified or undermodified tRNA $\mathrm{CAA}_{\mathrm{CAA}}$ leads to the suboptimal translation of $r p o S$ and iraP ORFs (Fig. 6). Suboptimal iraP levels lead to decreased rpoS stability, further decreasing steady-state levels of rpoS (Fig. 6).

\section{MATERIALS AND METHODS}

\section{Media and growth conditions}

M63-Glycerol-Sucrose-XG plates were used for positive selection of in-frame and out-of-frame $\mathrm{P}_{B A D}$-rpoS990-lacZ translational fusion (Court et al. 2003; Mandin and Gottesman 2010). Positive selection of in-frame and out-of-frame $\mathrm{P}_{B A D}$-rpoS990-lacZ translational fusions were confirmed by screening sucrose-resistant $\left(\mathrm{Suc}^{\mathrm{R}}\right)$ colonies for chloramphenicol-sensitivity $\left(\mathrm{Cm}^{\mathrm{S}}\right)$ on Luria-Bertani (LB) Lennox Agar plates supplemented with chloramphenicol to a final concentration of $25 \mu \mathrm{g} / \mathrm{mL}$. LB agar plates supplemented with zeomycin (LB-Zeo) or tetracycline (LB-Tet), to a final concentration of $25 \mu \mathrm{g} / \mathrm{mL}$, were used to select for transduction or recombineering of $r s s B:$ tet or $\triangle m i a A: z e o$ mutations, respectively. LB agar plates supplemented with ampicillin (LB-amp), to a final concentration of $100 \mu \mathrm{g} / \mathrm{mL}$ were used for selection of plasmids. All cultures for $\beta$-galactosidase assays were grown in LB Lennox media (KD Medical). LB Lennox media were supplemented with ampicillin to a final concentration of $100 \mu \mathrm{g} / \mathrm{mL}$ selection of pBR-leuX or pBR-leuZ plasmids. For arabinose induction experiments, cells were grown in LB Lennox containing $0.2 \%$ glucose (LB-Glu) to an $\mathrm{OD}_{600}$ of 1.0, washed with an equal volume of fresh LB Lennox to remove residual glucose, and resuspended in LB

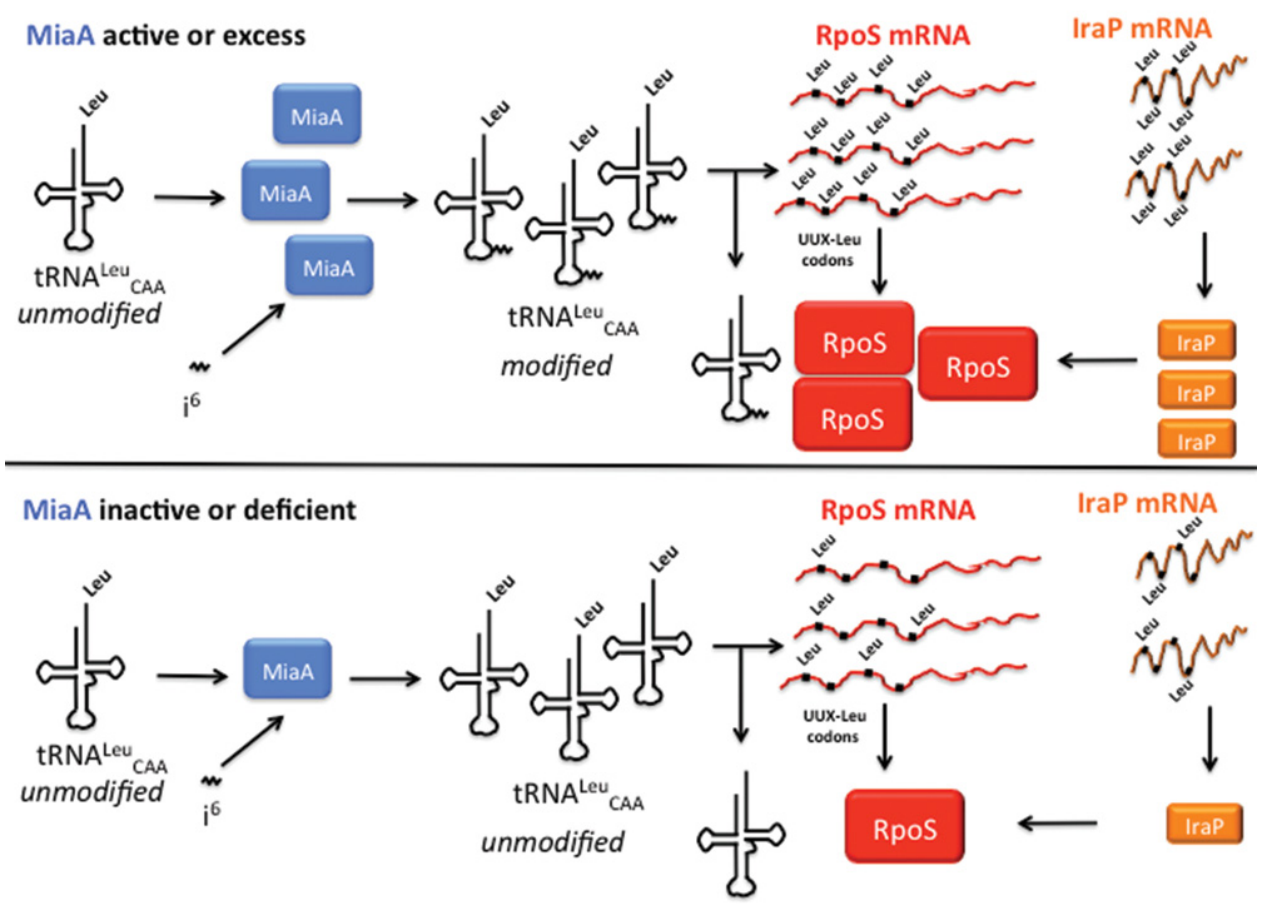

FIGURE 6. MiaA ( $\left.\mathrm{i}^{6} \mathrm{~A} 37\right)$ regulation of $r p o S$ and iraP model. This model illustrates our current model for the $\mathrm{i}^{6} \mathrm{~A} 37$ tuning of $r p o S$ and iraP expression. In the presence of excess and/or active MiaA, tRNA ${ }^{\text {leuX }}$ is $i^{6} \mathrm{~A} 37$ modified, leading to optimal translation of UXX-Leucine codons with both $r p o S$ and iraP. Since iraP increases $r p o S$ stability, optimal amounts of iraP contribute to increased steady-state levels of $r p o S$. When MiaA is inactive or limiting, tRNA $^{\text {leuX }}$ is unmodified, leading to suboptimal translation of UXX-Leucine codons within both rpoS and iraP. Suboptimal translation of iraP will result in decreased stability of rpoS. These combined factors significantly decrease the steady-state levels of rpoS. 
Lennox with $0.2 \%$ arabinose. Upon shifting cultures to LB-Ara, aliquots of the cultures were taken periodically following arabinose induction for $\beta$-galactosidase assays.

\section{Strains and plasmids}

Strains and plasmids are listed in Supplemental Table S1. All $\mathrm{P}_{B A D^{-}}$ rpoS990-lacZ translational fusion strains used for the experiments carried a mutation in $r s s B, r s s B:$ :tet, to rule out possible effects on rpoS stability.

\section{Construction of $P_{\mathrm{BAD}}$-rpoS990-lacZ translational fusions}

Arabinose-inducible translational fusions of rpoS990 to lacZ were constructed by recombineering strain PM1805. Strain PM1805 contains the $\lambda$-Red proteins under control of the temperature-sensitive allele of the $\lambda$-repressor, cI857. PM1805 also contains the counter-selectable marker, $c a t-s a c B$, at the lac locus to allow for positive selection of recombinant fusions in the presence of sucrose. Recombineering into PM1805 requires induction of the $\lambda$-Red proteins, creation of electrocompetent cells, electroporation of allelic exchange substrates, and positive selection of fusions in the presence of sucrose. To induce the $\lambda$-Red proteins, PM1805 was grown in $\mathrm{LB}$ at $32^{\circ} \mathrm{C}$ to $\mathrm{OD}_{600}$ of 0.5 and shifted to $43.5^{\circ} \mathrm{C}$ for $15 \mathrm{~min}$. The culture was then cooled in an ice-water bath. Then, washing the induced PM1805 culture in ice-cold $\mathrm{H}_{2} \mathrm{O}$ made electrocompetent cells. Approximately $100 \mathrm{ng}$ of the purified PCR product corresponding to the allelic exchange substrates were electroporated into the electrocompetent-induced PM1805 using the GenePulser (BioRad) on the Ec1 setting. Electroporated cells were recovered overnight in $10 \mathrm{~mL}$ of $\mathrm{LB}$ (Lennox) broth in a $125 \mathrm{~mL}$ flask at $32^{\circ} \mathrm{C}$. Overnight cultures were serially diluted and spread on M63-Glycerol-Sucrose-XG plates and incubated at $30^{\circ} \mathrm{C}$ for $3-5 \mathrm{~d}$. The purified PCR products were electroporated into electrocompetent PM1805. For construction of the wild-type $\mathrm{P}_{B A D^{-}}$rpoS990-lac $Z$ translation fusion, an allelic exchange substrate was created through the amplification of a portion of the $\mathrm{P}_{B A D^{-}}$ rpoS990-lacZ translational fusion from genomic DNA of strain KMT581 using oligonucleotide primers KT1123 and KT1124 (Table 2).

Three different derivatives of the $\mathrm{P}_{B A D^{-}}$rpoS990-lacZ translation fusion were also constructed: $\mathrm{P}_{B A D^{-}}$rpoS990(leu*1)-lac $Z, \mathrm{P}_{B A D^{-}}$ rpoS990(leu*2)-lacZ, $\mathrm{P}_{B A D^{-}} r p o S 990\left(l e u^{*} 3\right)$-lacZ translational fusions. The $\mathrm{P}_{B A D^{-}}$rpoS990(leu*1)-lacZ contained UUA to CUU changes across the entire ORF. The $\mathrm{P}_{B A D^{-}}$rpoS990(leu*2)-lac $Z$ contained UUG to CUC changes across the entire ORF. The $\mathrm{P}_{B A D^{-}}$rpoS990(leu*3)-lacZ contained both UUA to CUU and UUG to CUC changes across the entire ORF, effectively removing all MiaA-sensitive leucine codons. For the $\mathrm{P}_{B A D^{-r p o S} 990}$ (leu*1)-lacZ $\mathrm{P}_{B A D^{-}}$rpoS990(leu*2)-lacZ translational fusions, synthetic (gBlock) DNA fragments (IDT Technologies) " $\mathrm{P}_{B A D^{-}}$ rpoS990(leu*1)-lacZ_AES" and "P $\mathrm{P}_{B A D}$-rpoS990(leu*2)-lacZ_AES" (Supplemental Table S2), respectively, were PCR amplified using oligonucleotide primers KT1123 and KT1124 and used as an AES for recombineering into electrocompetent PM1800. For the $\mathrm{P}_{B A D}$-rpoS990(leu*3)-lac $Z$ translational fusions, two synthetic (gBlock) DNA fragments (IDT Technologies) with overlapping homology, "P ${ }_{B A D^{-}}$rpoS990(MiaA_Leu-) gBlockup" and " $\mathrm{P}_{B A D^{-}}$ rpoS990(MiaA_Leu-) gBlockdown” (Supplemental Table S2), were recombined together using the Gibson Assembly Mastermix
(New England Biolabs). The resulting DNA fragment was PCR amplified using oligonucleotide primers KT1123 and KT1124 (Table 2) and used as an AES for recombineering into electrocompetent PM1805.

\section{Construction of $\Delta$ miaA::zeo allele by recombineering}

The miaA gene was deleted and replaced with a zeomycin (zeo) resistance cassette by recombineering in strain KMT194. An allelic exchange substrate for miaA mutagenesis was created by PCR amplification of the zeo cassette from genomic DNA from strain KMT465, using oligonucleotide primers KT1035 and KT1036 (Table 2). The PCR product was purified and $\sim 100 \mathrm{ng}$ was used for electroporation into electrocompetent KMT194 after induction of the $\lambda$-Red proteins, using a protocol identical to the one used to induce the $\lambda$-Red proteins in, and prepare electrocompetent cells of, PM1805. After electroporation, putative recombinants carrying $\Delta$ miaA::zeo mutations were selected on LB-Zeo plates. Recombinants were purified once on LB-Zeo plates and twice on LB plates. The $\triangle$ miaA::zeo mutation was confirmed by PCR and phenotypic analysis.

\section{Construction of $P_{\mathrm{BAD}}$-iraP258-lacZ translational fusions}

We constructed arabinose-inducible translational fusions of the entire iraP ORF, except for the termination codon iraP258, to lacZ by recombineering strain PM1805. The methods used were identical to those listed in the construction of the $P_{B A D}$-iraP258-lac $Z$ translational fusion strains. We also created three different derivatives of the $\mathrm{P}_{B A D}$-iraP258-lac $Z$ translation fusion in a manner identical to the rpoS fusions: $\mathrm{P}_{B A D^{-}}$iraP258(leu*1)-lac $Z, \mathrm{P}_{B A D^{-}}$-iraP258(leu*2)-

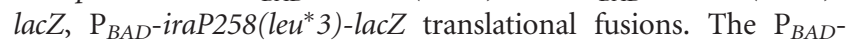
iraP258(leu*1)-lac $Z$ contained UUA to CUU changes across the entire ORF. The $\mathrm{P}_{B A D}$-iraP258(leu*2)-lac $Z$ contained UUG to CUU changes across the entire ORF. The $\mathrm{P}_{B A D}$-iraP258(leu*3)-lacZ translational fusion contained both UUA to CUU and UUG to CUU changes across the entire iraP ORF, effectively removing all MiaAsensitive leucine codons from the iraP portion of the fusion. For

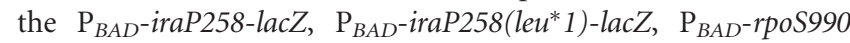

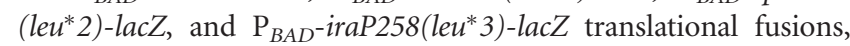
synthetic (gBlock) DNA fragments (IDT Technologies) " $\mathrm{P}_{B A D^{-}}$

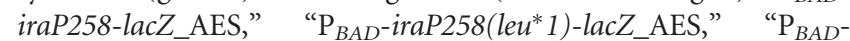
rpoS990(leu*2)-lacZ_AES," and "P $\mathrm{P}_{B A D}$-iraP258(leu*3)-lacZ AES" (Supplemental Table S2), respectively, were PCR amplified using oligonucleotide primers KT1162 and KT1163 and used as an AES for recombineering into electrocompetent PM1800.

\section{P1 transduction to moved marked mutations}

All $\triangle r s s B:$ tet and $\triangle m i a A:: z e o$ mutations were transferred into the

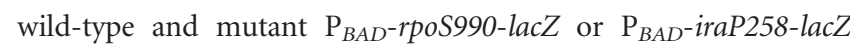
translational fusion strains by bacteriophage $\mathrm{P} 1$ transduction and selecting for tetracycline $\left(\mathrm{Tet}^{\mathrm{R}}\right)$ or zeomycin $\left(\mathrm{Zeo}^{\mathrm{R}}\right)$ resistance, respectively.

\section{DNA manipulations and cloning reactions}

To determine whether overexpression of rare leucine tRNAs could suppress the MiaA requirement for rpoS expression, we cloned rare leucine tRNA genes, leuX and leuZ, downstream from the 
IPTG-inducible $\mathrm{P}_{\text {Llaco }}$ promoter in plasmid pBR-pLac (Guillier and Gottesman 2006).

\section{Construction of $p B R$-leuX}

The leuX gene was amplified from E. coli K12 MG1655 chromosomal DNA using oligonucleotide primers KT1104 and KT1105 (Table 2). The PCR product was then purified using the PCR Purification Kit (Lamda Biotech), digested with restriction enzymes AatII (New England Biolabs) and EcoRI (New England Biolabs), and ligated to an AatII/EcoRI digest of plasmid pBR-pLac (Supplemental Table S1) using Quick Ligase (New England Biolabs). An aliquot of the ligation reaction was transformed into NEB5 chemically competent cells (New England Biolabs) using heat shock transformation at $42^{\circ} \mathrm{C}$ for $30 \mathrm{sec}$, recovered in $500 \mu \mathrm{L}$ SOC Media, and $100 \mu \mathrm{L}$ aliquot was spread on LB-Amp plates. Putative pBR-leuX transformants were screened by colony PCR using the BAC-Direct Kit and oligonucleotide primers KT1115 and KT1105. Plasmids from PCR positive transformants were sequenced to confirm the presence of the leuX insert. Plasmid pBR-leuX was transformed into wild-type and $\triangle$ miaA::zeo fusion strain using TSS transformation (Chung et al. 1989). The clones were confirmed by DNA sequencing.

\section{Construction of $p B R-l e u Z$}

The steps used for construction of pBR-leuZ are identical to those used for the construction of pBR-leuX with the exception of the oligonucleotide primers used for PCR amplification (oligonucleotide primers KT1102 and KT1103) and colony PCR screening (oligonucleotide primers KT1115 and KT1103). The clones were confirmed by DNA sequencing.

\section{$\beta$-galactosidase assays}

High-throughput kinetic $\beta$-galactosidase assays were carried out in 96-well plates as previously described (Zhou and Gottesman 1998). The Filtermax F5 (Molecular Devices) multimode microplate reader was used to read microtiter plates. $\beta$-galactosidase-specific activity units are defined as the slope of $\mathrm{OD}_{420}$ reading divided by $\mathrm{OD}_{600}$ and are approximately 25 -fold lower than Miller Units.

\section{Experimental design for assays executed following} arabinose induction

Briefly, samples to be assayed were grown in $5 \mathrm{~mL}$ of LB-Glu overnight at $37^{\circ} \mathrm{C}$ in a roller drum. Overnight cultures were diluted $1: 1000$ in $30 \mathrm{~mL}$ of fresh LB-Glu in a $125-\mathrm{mL}$ Erlenmeyer flask and grown at $37^{\circ} \mathrm{C}$ in a shaking water bath at $200 \mathrm{rpm}$. When cultures reached an $\mathrm{OD}_{600}$ of 1.0, cells were harvested by centrifugation and resuspended in $30 \mathrm{~mL}$ of fresh $\mathrm{LB}$ supplemented $0.2 \%$ arabinose, and $100 \mu \mathrm{L}$ aliquots of each culture were taken every $5 \mathrm{~min}$ for $\beta$-galactosidase assays. Samples were collected in triplicate for each individual experiment and averages were taken as a representative sample for each experiment. The data represent the mean and standard error of the mean of at least three independent replicates.

\section{SUPPLEMENTAL MATERIAL}

Supplemental material is available for this article.

\section{ACKNOWLEDGMENTS}

We thank Rich Maraia, Patrick Thiaville, Kelly T. Hughes, Kumaran Ramamurthi, Nadim Majdalani, Gisela Storz, and Susan Gottesman for comments on and critical analysis of this manuscript. We also thank Patrick Thiaville and Valerie de Crecy-Lagard for providing us with a table of leu codon usage for each ORF in the E. coli genome, based on the formerly active Codon Counting Database from Tom Begley. This research was supported by the National Institute of General Medical Sciences of the National Institutes of Health under award number SC2 GM105419 (K.M.T.). This research was also supported by the National Science Foundation under the LSAMP program (HRD-1000286 [M.O.]). Any opinions, findings, conclusions, or recommendations expressed in this material are those of the author(s) and do not necessarily reflect the views of the National Institutes of Health or the National Science Foundation.

Received October 7, 2015; accepted February 5, 2016.

\section{REFERENCES}

Bartz JK, Kline LK, Söll D. 1970. $\mathrm{N}^{6}-\left(\Delta^{2}\right.$-isopentenyl $)$ adenosine: biosynthesis in vitro in transfer RNA by an enzyme purified from Escherichia coli. Biochem Biophys Res Commun 40: 1481-1487.

Battesti A, Majdalani N, Gottesman S. 2015. Stress sigma factor RpoS degradation and translation are sensitive to the state of central metabolism. Proc Natl Acad Sci 112: 5159-5164.

Begley TJ, Dyavaiah M, Patil A, Rooney JP, Direnzo D, Young CM, Conklin DS, Zitomer RS, Begley TJ. 2007. Trm9-catalized tRNA modifications link translation to the DNA damage response. Mol Cell 28: 860-870.

Björk GR, Durand JM, Hagervall TG, Leipuviene R, Lundgren HK, Nilsson K, Chen P, Qian Q, Urbonavicius J. 1999. Transfer RNA modification: influence on translational frameshifting and metabolism. FEBS Lett 452: 47-51.

Blum PH. 1988. Reduced leu operon expression in a miaA mutant of Salmonella typhimurium. J Bacteriol 170: 5125-5133.

Bougdour A, Wickner S, Gottesman S. 2006. Modulating RssB activity: IraP, a novel regulator of $\mathrm{s}^{\mathrm{S}}$ stability in Escherichia coli. Genes Dev 20: 884-897.

Bougdour A, Cunning C, Baptiste PJ, Elliott T, Gottesman S. 2008. Multiple pathways for regulation of $\sigma^{\mathrm{S}}$ (RpoS) stability in Escherichia coli via the action of multiple anti-adaptors. Mol Microbiol 68: 298-313.

Brown L, Elliot T. 1996. Efficient translation of the RpoS sigma factor in Salmonella typhimurium requires host factor I, an RNA-binding protein encoded by the $h f q$ gene. J Bacteriol 178: 3763-3770.

Chan CT, Dyavaiah M, DeMott MS, Taghizadeh K, Dedon PC, Begley TJ. 2010. A quantitative system approach reveals dynamic control of tRNA modifications during cellular stress. PLoS Genet 6: e1001247.

Chan CT, Pang YL, Deng W, Babu IR, Dyavaiah M, Begley TJ, Dedon PC. 2012. Reprogramming of tRNA modifications controls the oxidative stress response by codon-biased translation of proteins. Nat Commun 3: 937.

Chen CC, Fang M, Majumder A, Wu HY. 2001. A 72-base pair AT-rich DNA sequence element functions as a bacterial gene silencer. J Biol Chem 276: 9478-9485.

Chung CT, Niemela SL, Miller RH. 1989. One-step preparation of competent Escherichia coli: transformation and storage of bacterial cells in the same solutions. Proc Natl Acad Sci 86: 2172-2175.

Court DL, Swaminathan S, Yu D, Wilson H, Baker T, Bubunenko M, Sawitzke J, Sharan SK. 2003. Mini- $\lambda$ : a tractable system for chromosome and BAC engineering. Gene 315: 63-69. 
Deutsch C, El Yacoubi B, de Crecy-Lagard V, Iwata-Reuyl D. 2012. Biosynthesis of threonylcarbamoyl adenosine $\left(\mathrm{t}^{6} \mathrm{~A}\right)$, a universal tRNA nucleoside. J Biol Chem 287: 13666-13673.

Durand JM, Björk GR, Kuwae A, Yoshikawa M, Sasakawa C. 1997. The modified nucleoside 2-methylthio- $N^{6}$-isopentenyladenosine in tRNA of Shigella flexneri is required for expression of virulence genes. J Bacteriol 179: 5777-5782.

Durand JM, Dagberg B, Uhlin BE, Björk GR. 2000. Transfer RNA modification, temperature and DNA superhelicity have a common target in the regulatory network of the virulence of Shigella flexneri: the expression of the virF gene. Mol Microbiol 35: 924-935.

Endres L, Dedon PC, Begley TJ. 2015. Codon-biased translation can be regulated by wobble-base tRNA modification systems during cellular stress responses. RNA Biol 12: 603-614.

Ernsting BR, Atkinson MR, Ninfa AJ, Matthews RG. 1992. Characterization of the regulon controlled by the leucine-responsive regulatory protein in Escherichia coli. J Bacteriol 174: 1109-1118.

Esberg B, Leung HC, Tsui HC, Bjork GR, Winkler ME. 1999. Identification of the miaB gene, involved in methylthiolation of isopentenylated A37 derivatives in the tRNA of Salmonella typhimurium and Escherichia coli. J Bacteriol 181: 7256-7265.

Golovko A, Hjälm G, Sitbon F, Nicander B. 2000. Cloning of a human tRNA isopentenyl transferase. Gene 258: 85-93.

Gottesman S. 1996. Proteases and their targets in Escherichia coli. Annu Rev Genet 30: 465-506.

Gowrishankar J, Pittard J. 1982. Regulation of phenylalanine biosynthesis in Escherichia coli K-12: control of transcription of the pheA operon. J Bacteriol 150: 1130-1137.

Gray J, Wang J, Gelvin SB. 1992. Mutation of the miaA gene of Agrobacterium tumefaciens results in reduced vir gene expression. J Bacteriol 174: 1086-1098.

Guillier M, Gottesman S. 2006. Remodelling of the Escherichia coli outer membrane by two small regulatory RNAs. Mol Microbiol 59: 231247.

Hengge-Aronis R. 1993. Survival of hunger and stress: the role of rpoS in early stationary phase gene regulation in E. coli. Cell 72: $165-168$.

Hengge-Aronis R. 1996. Back to log phase: $\sigma^{\mathrm{S}}$ as a global regulator in the osmotic control of gene expression in Escherichia coli. Mol Microbiol 21: $887-893$.

Hirsch M, Elliott T. 2002. Role of ppGpp in rpoS stationary-phase regulation in Escherichia coli. J Bacteriol 184: 5077-5087.

Klauck E, Böhringer J, Hengge-Aronis R. 1997. The LysR-like regulator $\mathrm{LeO}$ in Escherichia coli is involved in the translational regulation of rpos by affecting the expression of the small regulatory DsrARNA. Mol Microbiol 25: 559-569.

Kolmsee T, Hengge R. 2011. Rare codons play a positive role in the expression of the stationary phase sigma factor RpoS $\left(\sigma^{\mathrm{S}}\right)$ in Escherichia coli. RNA Biol 8: 913-921.

Lamichhane TN, Blewett NH, Crawford AK, Cherkasova VA, Iben JR, Begley TJ, Farabaugh PJ, Maraia RJ. 2013a. Lack of tRNA modification isopentenyl-A37 alters mRNA decoding and causes metabolic deficiencies in fission yeast. Mol Cell Biol 33: 2918-2929.

Lamichhane TN, Mattijssen S, Maraia RJ. 2013b. Human cells have a limited set of tRNA anticodon loop substrates of the tRNA isopentenyltransferase TRIT1 tumor suppressor. Mol Cell Biol 33: 49004908.

Landick R, Yanofsky C, Choo K, Phung L. 1990. Replacement of the Escherichia coli trp operon attenuation control codons alters operon expression. J Mol Biol 216: 25-37.

Lange R, Fischer D, Hengge-Aronis R. 1995. Identification of transcriptional start sites and the role of ppGpp in the expression of rpoS, the structural gene for the $\sigma^{\mathrm{S}}$ subunit of RNA polymerase in Escherichia coli. J Bacteriol 177: 4676-4680.

Lin R, D'Ari R, Newman EB. 1992. $\lambda$ placMu insertions in genes of the leucine regulon: extension of the regulon to genes not regulated by leucine. J Bacteriol 174: 1948-1955.
Majdalani N, Cunning C, Sledjeski DD, Elliott T, Gottesman S. 1998. DsrA RNA regulates translation of RpoS message by an anti-antisense mechanism, independent of its action as an antisilencer of transcription. Proc Natl Acad Sci 95: 12462-12467.

Majdalani N, Hernandez D, Gottesman S. 2002. Regulation and mode of action of the second small RNA activator of RpoS translation, RprA. Mol Microbiol 46: 813-826.

Mandin P, Gottesman S. 2010. Integrating anaerobic/aerobic sensing and the general stress response through the ArcZ small RNA. EMBO J 29: 3094-3107.

Martin NC, Hopper AK. 1982. Isopentenylation of both cytoplasmic and mitochondrial tRNA is affected by a single nuclear mutation. J Biol Chem 257: 10562-10565.

Pages D, Buckingham RH. 1990. Mutants of pheV in Escherichia coli affecting control by attenuation of the pheS, $T$ and pheA operons. Two distinct mechansims for de-attenuation. J Mol Biol 216: $17-24$.

Patil A, Dyavaiah M, Joseph F, Rooney JP, Chan CT, Dedon PC, Begley TJ. 2012. Increased tRNA modification and gene-specific codon usage regulate cell cycle progression during the DNA damage response. Cell Cycle 1: 3656-3665.

Pettersson BM, Kirsebom LA. 2011. tRNA accumulation and suppression of the bldA phenotype during development in Streptomyces coelicolor. Mol Microbiol 79: 1602-1614.

Platko JV, Calvo JM. 1993. Mutations affecting the ability of Escherichia coli Lrp to bind DNA, activate transcription, or respond to leucine. J Bacteriol 175: 1110-1117.

Ranquet C, Gottesman S. 2007. Translational regulation of the Escherichia coli stress factor RpoS: a role for SsrA and Lon. J Bacteriol 189: 4872-4879.

Repoila F, Gottesman S. 2001. Signal transduction cascade for regulation of RpoS: temperature regulation of DsrA. J Bacteriol 183: 4012-4023.

Sledjeski DD, Gupta A, Gottesman S. 1996. The small RNA, DsrA, is essential for the low temperature expression of RpoS during exponential growth in Escherichia coli. EMBO J 15: 3993-4000.

Spinola M, Galvan A, Pignatiello C, Conti B, Pastorino U, Nicander B, Paroni R, Dragani TA. 2005. Identification and functional characterization of the candidate tumor suppressor gene TRIT1 in human lung cancer. Oncogene 24: 5502-5509.

Spinola M, Falvella FS, Galvan A, Pignatiello C, Leoni VP, Pastorino U, Paroni R, Chen S, Skaug V, Haugen A, et al. 2007. Ethnic differences in frequencies of gene polymorphisms in the MYCL1 region and modulation of lung cancer patients' survival. Lung Cancer 55: 271-277.

Stratmann T, Pul Ü, Wurm R, Wagner R, Schnetz K. 2012. RcsB-BglJ activates the Escherichia coli leuO gene, encoding an $\mathrm{H}-\mathrm{NS}$ antagonist and pleiotropic regulator of virulence determinants. Mol Microbiol 83: 1109-1123.

Tanaka K, Takayanagi Y, Fujita N, Ishihama A, Takahashi H. 1993. Heterogeneity of the principal sigma factor in Escherichia coli: the $r p o S$ gene product, $\sigma^{38}$, is a second principal $\sigma$ factor of RNA polymerase in stationary-phase Escherichia coli. Proc Natl Acad Sci 90: 8303.

Thompson KM, Gottesman S. 2014. The MiaA tRNA modification enzyme is necessary for robust RpoS expression in Escherichia coli. J Bacteriol 196: 754-761.

Tsui HC, Winkler ME. 1994. Transcriptional patterns of the mutL-miaA superoperon of Escherichia coli K-12 suggest a model for posttranscriptional regulation. Biochimie 76: 1168-1177.

Tsui HC, Leung HC, Winkler ME. 1994. Characterization of broadly pleiotropic phenotypes caused by an $h f q$ insertion mutation in Escherichia coli K-12. Mol Microbiol 13: 35-49.

Urbonavicius J, Qian Q, Durand JM, Hagervall TG, Björk GR. 2001. Improvement of reading frame maintenance is a common function for several tRNA modifications. EMBO $J$ 20: 48634873.

Urbonavicius J, Stahl G, Durand JM, Ben Salem SN, Qian Q, Farabaugh P, Björk GR. 2003. Transfer RNA modifications that 


\section{Aubee et al.}

alter +1 frameshifting in general fail to affect -1 frameshifting. $R N A$ 9: 760-768.

Vold BS, Lazar JM, Gray AM. 1979. Characterization of a deficiency of $N^{6}$-( $\Delta^{2}$-isopentenyl)-2-methylthioadenosine in the Escherichia coli mutant $\operatorname{trp} X$ by use of antibodies to $N^{6}-\left(\Delta^{2}\right.$-isopentenyl $)$ adenosine. J Biol Chem 254: 7362-7367.

Winkler ME. 1998. Genetics and regulation of base modification in the tRNA and rRNA of prokaryotes and eukaryotes. In Modification and editing of RNA (ed. Grosjean H, Benne R), pp. 441-469. ASM Press, Washington, DC.

Yarham JW, Lamichhane TN, Pyle A, Mattijssen S, Baruffini E, Bruni F, Donnini C, Vassilev A, He L, Blakely EL, et al. 2014. Defective $i^{6} \mathrm{~A} 37$ modification of mitochondrial and cytosolic tRNAs results from pathogenic mutations in TRIT1 and its substrate tRNA. PLoS Genet 10: e1004424.

Zambrano MM, Siegele DA, Almirón M, Tormo A, Kolter R. 1993. Microbial competition: Escherichia coli mutants that take over stationary phase cultures. Science 259: 1757-1760.

Zhang W, Collinet B, Perrochia L, Durand D, van Tilbeurgh H. 2015. The ATP-mediated formation of the YgjD-YeaZ-YjeE complex is required for the biosynthesis of tRNA $t^{6} \mathrm{~A}$ in Escherichia coli. Nucleic Acids Res 43: 1804-1817.

Zhou Y, Gottesman S. 1998. Regulation of proteolysis of the stationaryphase sigma factor RpoS. J Bacteriol 180: 1154-1158.

Zinser ER, Kolter R. 2000. Prolonged stationary-phase incubation selections for lrp mutations Escherichia coli K-12. J Bacteriol 182: 4361-4365. 

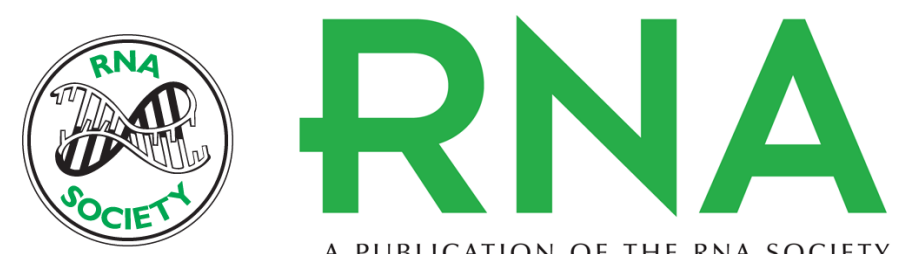

A PUBLICATION OF THE RNA SOCIETY

\section{The $i^{6}$ A37 tRNA modification is essential for proper decoding of UUX-Leucine codons during rpoS and iraP translation}

Joseph I. Aubee, Morenike Olu and Karl M. Thompson

RNA 2016 22: 729-742 originally published online March 15, 2016

Access the most recent version at doi:10.1261/rna.053165.115

\section{Supplemental http://rnajournal.cshlp.org/content/suppl/2016/03/02/rna.053165.115.DC1 Material}

References This article cites 60 articles, 30 of which can be accessed free at: http://rnajournal.cshlp.org/content/22/5/729.full.html\#ref-list-1

Creative This article is distributed exclusively by the RNA Society for the first 12 months after the Commons License full-issue publication date (see http://rnajournal.cshlp.org/site/misc/terms.xhtml). After 12 months, it is available under a Creative Commons License (Attribution-NonCommercial 4.0 International), as described at http://creativecommons.org/licenses/by-nc/4.0/.
Email Alerting Receive free email alerts when new articles cite this article - sign up in the box at the Service top right corner of the article or click here.

\section{III!" PI Providing Precise Solutions for your research.}

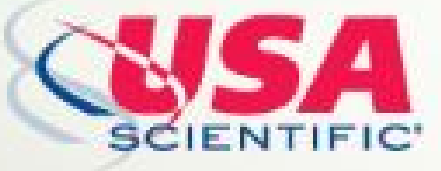

To subscribe to $R N A$ go to:

http://rnajournal.cshlp.org/subscriptions

(C) 2016 Aubee et al.; Published by Cold Spring Harbor Laboratory Press for the RNA Society 